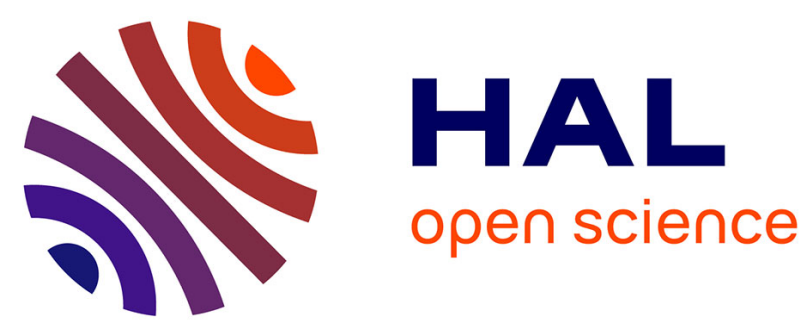

\title{
Experimental investigation of spray impingement hydrodynamic on a hot surface at high flow rates using phase Doppler analysis and infrared thermography
}

Alexandre Labergue, Michel Gradeck, Fabrice Lemoine

\section{To cite this version:}

Alexandre Labergue, Michel Gradeck, Fabrice Lemoine. Experimental investigation of spray impingement hydrodynamic on a hot surface at high flow rates using phase Doppler analysis and infrared thermography. International Journal of Heat and Mass Transfer, 2016, 100, pp.65 - 78. 10.1016/j.ijheatmasstransfer.2016.01.041 . hal-01580485

\section{HAL Id: hal-01580485 \\ https://hal.science/hal-01580485}

Submitted on 1 Sep 2017

HAL is a multi-disciplinary open access archive for the deposit and dissemination of scientific research documents, whether they are published or not. The documents may come from teaching and research institutions in France or abroad, or from public or private research centers.
L'archive ouverte pluridisciplinaire HAL, est destinée au dépôt et à la diffusion de documents scientifiques de niveau recherche, publiés ou non, émanant des établissements d'enseignement et de recherche français ou étrangers, des laboratoires publics ou privés. 


\title{
Experimental investigation of spray impingement hydrodynamic on a hot surface at high flow rates using phase Doppler analysis and infrared thermography
}

\author{
A. Labergue ${ }^{1,2}$, M. Gradeck ${ }^{1,2}$, F. Lemoine ${ }^{1,2}$ \\ ${ }^{1}$ LEMTA, UMR 7563, Université de Lorraine, Vandoeuvre-Lès-Nancy, 54518, \\ France \\ ${ }^{2}$ LEMTA, UMR 7563, CNRS, Vandoeuvre-Lès-Nancy, 54518, France
}

\begin{abstract}
Large panels of studies have been performed in order to investigate the dynamic behavior of a droplet during the impingement onto a rigid wall. Most of them are dealing with single droplets and only fewer are devoted to sprays, for which several diagnostics were implemented. Moreover, when sprays are involved, the liquid mass flux is generally quite low (on the order of several $\mathrm{kg} / \mathrm{m}^{2} / \mathrm{s}$ ) and the case of a cold surface was mainly considered. Therefore, the present experimental work aims at investigating the impingement of sprays onto hot surfaces (up to $800^{\circ} \mathrm{C}$ ) with liquid mass flux up to $13 \mathrm{~kg} / \mathrm{m}^{2} / \mathrm{s}$. Specifically, the main objective is to characterize the secondary droplets as a function of the surface temperature, starting from the Leidenfrost regime. To that purpose, a 2D Phase Doppler Analyzer (PDA) is synchronized with an infrared camera in order to measure simultaneously the time evolution of the local droplets size and velocity distributions with the surface temperature. Six full cone sprays were used in order to obtain a wide range of impingement conditions (normal incident Weber and liquid mass flux). A comparison of the impingement characteristics is also performed when the surface remains at room temperature. Another important point concerns also the effect on the spray flow of the presence of the surface. Results show clearly that the incident droplet trajectories are modified by the presence of the solid wall itself and depend strongly on the temperature surface. The in-depth investigation of the impingement characteristics of the spray is mainly focused on the description of the statistical mean diameter and the mass flux after the impingement. The main results highlight that the mean diameter value of the secondary droplets does not change during the Leidenfrost regime and increases for lower surface temperature. This behavior is strongly correlated to the liquid film formation for temperatures lower than the Leidenfrost point. The expelled mass flux exhibits a similar behavior but a decrease is observed after a given temperature, which can be attributed to the deepening of the liquid, leading to a reduction of the number of expelled droplets.
\end{abstract}

Keywords: Spray cooling; phase Doppler measurements; infrared thermography; spray impinge-

ment.

Nomenclature:

$\begin{array}{lll}\text { We } & \text { Weber number } & (-) \\ \text { Oh } & \text { Ohnesorge number } & (-) \\ K & \text { Mundo number } & (-) \\ C_{p} & \text { specific capacity } & (\mathrm{J} / \mathrm{Kg} / \mathrm{K}) \\ a & \text { thermal diffusivity } & \mathrm{m} / \mathrm{s}^{2} \\ k & \text { thermal conductivity } & \mathrm{W} / \mathrm{m} / \mathrm{K} \\ R & \text { disk radius } & (\mathrm{m}) \\ e & \text { disk thickness } & (\mathrm{m}) \\ D & \text { droplet diameter } & (\mathrm{m})\end{array}$




$\begin{array}{cll}h & \text { enthalpy } & (\mathrm{J} / \mathrm{kg}) \\ G & \text { liquid mass flux } & \left(\mathrm{kg} / \mathrm{m}^{2} / \mathrm{s}\right) \\ q^{\prime \prime} & \text { heat flux density } & \left(\mathrm{W} / \mathrm{m}^{2}\right) \\ v, w & \text { droplet velocity component } & (\mathrm{m} / \mathrm{s}) \\ r & \text { radial coordinate } & (\mathrm{m}) \\ T & \text { temperature } & (\mathrm{K}) \\ t & \text { time } & (\mathrm{s})\end{array}$

\section{Greel symbols}

$\begin{array}{lll}\alpha & \text { spray angle } & \left({ }^{\circ}\right) \\ \lambda & \text { fractions } & (-) \\ \rho & \text { mass density } & \left(\mathrm{kg} / \mathrm{m}^{3}\right) \\ \mu & \text { dynamic viscosity } & (\mathrm{Pa} . \mathrm{s}) \\ \sigma & \text { surface tension } & (\mathrm{N} / \mathrm{m})\end{array}$

\section{Symbols}

$\begin{array}{ll}* & \text { dimensionless } \\ \sim & \text { time average } \\ - & \text { arithmetic mean }\end{array}$

\section{Subscripts}

$a$ after impact

$b$ before impact

$d$ relative to single droplet

$l$ property of liquid phase

$n$ normal to the surface

Leid Leidenfrost temperature

sat liquid saturation properties

sub surface subcooling

$v$ property of vapor phase

$w$ conditions at the disk surface

$m$ mass

$N$ number

10 mean diameter

32 Sauter diameter

$\mathrm{Ni}$ relative to the Nickel properties

\section{Introduction}

Studies of spray-wall interactions have always received a lot of attention due to the numerous industrial processes involving the impingement of droplets onto a solid wall. In some cases, the wall is heated like in a combustion chamber of a direct injection engine or for applications where the dissipation of high heat flux is required (steel industry, cooling of electronics components or nuclear safety issues). In other cases, the surface temperature is close to the liquid one and no heat transfer occurs; it concerns applications such as spray coating or spray painting. In both cases, the hydrodynamics of the impingement is mainly characterized by the generation of the secondary spray (i.e. after impact) and the potential formation of a liquid film on the surface. The latter depends on several parameters such as the surface temperature, the relative roughness of the surface and also on the incident droplet properties (viscosity, density, surface tension, diameter, velocity and incident impact angle). Usually, two main dimensionless parameters are used to describe the impact conditions: the Weber and Ohnesorge numbers respectively defined by $W e=\rho U^{2} D / \sigma$ and $O h$ $=\mu /(\rho D \sigma)^{1 / 2}$ where $U$ and $D$ are the velocity and the diameter of the droplet before impact, $\mu$, $\rho$ and $\sigma$ 
are the dynamic viscosity, the mass density and the surface tension respectively. In addition, in order to account for the influence of the surface temperature, a dimensionless temperature $T^{*}$ is introduced:

$$
T^{*}=\frac{T_{w}-T_{\text {sat }}}{T_{\text {Leid }}-T_{\text {sat }}}
$$

where $T_{w}, T_{\text {sat }}$ and $T_{\text {Leid }}$ are respectively the surface temperature, the saturation temperature of the liquid and the Leidenfrost temperature. The Leidenfrost regime $\left(T^{*}>1\right)$ is characterized by the presence of a vapor layer formed between the liquid and the solid wall, causing a dramatic reduction of the heat transfer. A cold impingement corresponds to a situation where $T^{*}<0$. Three main impingement regimes can be observed: rebound, splashing and deposition, the latter contributing to the formation of a liquid film. Introducing the Mundo number, $\mathrm{K}=\mathrm{WeOh}^{-0.4}[1]$ and using the dimensionless temperature $T^{*}$, it is possible to determine empirically the regime associated with a set of impact conditions. Compiling the results of Dewitte [2] and Cossali et.al [3] on a $K-T^{*}$ diagram for single droplets impinging onto a heated surface, the boundaries delimiting the different regimes can be outlined (Figure 1).

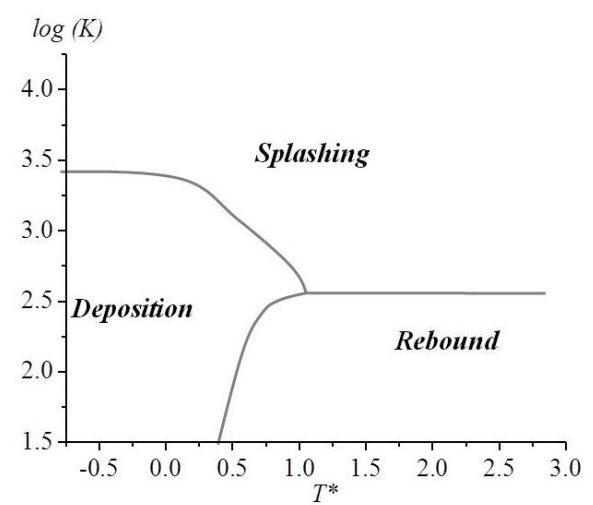

Figure 1: $K-T^{*}$ diagram representing the boundaries between the different impact regimes according to the dimensionless surface temperature and the Mundo number from the works [2-3].

Only rebound and splashing regimes can be observed in the Leidenfrost regime. For a narrow temperature range corresponding to $0.5<T^{*}<1$, the three regimes may exist. For $T^{*}<0.5$, according to the Mundo number value, an incoming droplet may deposit or splash on the surface.

Obviously, the characteristics of the impingement for a spray onto a solid surface are different compared to single droplets. On one hand, the boundaries in Figure 1, deduced from experiments conducted with single droplets, will more likely be different for sprays. On the other hand, as different droplet diameters or velocities are involved in a spray, the corresponding distribution of the Mundo number could lead to simultaneous different impact regimes for a given surface temperature. Consequently, the properties of the secondary droplets in the case of impinging sprays are expected be different compared to those of a single droplet [4-5].

Due to the multiples parameters and the complexity of the mechanisms of the droplets impingement, a large number of investigations are focused on single droplets impinging on a cold surface $[4 ; 8]$ or on a heated surface $[1-3 ; 9-18]$. On the contrary, works describing the secondary droplets in the case of spray impingement are more limited and concern mainly isothermal impingements [19-22]. In fact, studies dealing with sprays impinging onto heated surfaces are devoted to investigate the heat transfer between the liquid and the solid surface as a function of the initial sprays conditions: liquid mass flux or Weber number. In the particular case of a spray, two Weber numbers can be defined: (1) the usual single droplet Weber number $W e_{d}$ based on the diameter of a given droplet size class and the normal velocity of the corresponding class or (2) the mean Weber number $\overline{W e}$ based on the arithmetic mean droplet diameter and mean normal velocity. Moreover, a literature review reveals that the value of some operating parameters such as Weber number, liquid mass flux and wall temperature remain rela- 
tively low in comparison to standard industrial configurations. Al-Ahmadi [23] and Cox and Yao [24] have used conditions close to industrial operating conditions (droplet diameters up to several millimeters and liquid mass flux up to $30 \mathrm{~kg} / \mathrm{m}^{2} \cdot \mathrm{s}^{-1}$ respectively) but the focus of their work was on the estimation of the heat flux without any investigation of the secondary spray properties. Although Jia and Qiu [25] performed an investigation with a spray impinging on a heated surface in order to analyze the secondary droplet hydrodynamic as a function of the surface temperature, the liquid mass flux and the surface temperature did not exceed $1.2 \mathrm{~kg} / \mathrm{m}^{2} / \mathrm{s}$ and $160^{\circ} \mathrm{C}$ respectively.

The present paper follows a previous study conducted with water mass flux up to $8 \mathrm{~kg} / \mathrm{m}^{2} / \mathrm{s}$ [26]. The main purpose was to correlate the heat transfers observed at the solid wall with the spray properties. The aim of the present study is to analyze more thoroughly the mechanisms of the impingement by increasing the liquid mass flux up to $13.5 \mathrm{~kg} / \mathrm{m}^{2} / \mathrm{s}$, when the surface temperature is greater than the Leidenfrost one (about $800^{\circ} \mathrm{C}$ ). First, the new heat transfers results obtained with the higher liquid mass flux are presented. These first results are especially useful for the second part of the work devoted to the study of the impingement characteristics. Three main objectives are tackled: $i$ to compare the case of a cold impingement with the case of a heated surface, $i i$ - to analyze the influence of the presence of the solid surface on the droplets properties before impact and iii- to characterize the secondary spray as a function of the surface temperature. To reach these objectives, two synchronized experimental diagnostics were set-up: Phase Doppler measurement for the characterization of the droplets (diameter and velocity distributions) and an infrared thermography device to estimate both the heat flux removed by the droplets and the surface temperature. This paper will demonstrate the ability of the combination of these two devices as an innovative tool to analyze the properties of outgoing droplets induced by a spray impinging on a surface at high temperature.

\section{Experimental set-up and diagnostics}

\subsection{Spray facilities}

Figure 2 depicts the experimental facility specifically designed for investigating the spray impingement; this facility is similar to the one described in [26], where further details can be found. It consists in a closed loop to ensure the circulation of the coolant fluid (i.e. water). The surface to be cooled is a disk made of nickel disk (radius $R=85 \mathrm{~mm}$ and thickness $e=5 \mathrm{~mm}$ ). The spraying devices consist in full cone sprays and the coolant is water. Basically, an experiment is performed in two steps: the first one corresponds to the heating of the surface while the spray is turned-off and the second step corresponds to the cooling of the surface by applying the spray. In order to limit the establishment time of the spray flow (mainly due to the centrifugal pump start-up time), a second similar spray is flowing directly toward the main tank during the first step. Two piloted electrovalves allow to switch from one spray to the other one. The nickel disk is heated up by an electromagnetic induction system (power supply $12 \mathrm{~kW}$; Five-Celes ${ }^{\circledR}$ ). The disk is positioned on an insulating material and is centered just below the nozzle (A) along its main axis. The exit nozzle defines the origin of the $X-Y-Z$ axis.

\subsection{Phase Doppler Measurements}

The properties of the spray (velocity and droplets size) are measured by using a classic 2D phase Doppler system (PDA) manufactured by Dantec-Dynamics ${ }^{\circledR}$. The PDA excitation volume is generated by a LDA transmitter probe (Dantec-dynamics Fiber-Flow ${ }^{\circledR}$ probe; label (13)) coupled with an argon ion laser, operating in multiline mode (wavelengths of $514.5 \mathrm{~nm}$ and $488 \mathrm{~nm}$ ). The two wavelengths are used to achieve the measurements of the vertical and horizontal components of the droplet velocity vector (denoted by $w$ and $v$, along the $Z$ and $X$-axis respectively). In order to protect the PDA receiver (label (14)) against the expelled water after the impact on the solid surface, the receiver is tilted with an angle of $45^{\circ}$ from the solid surface plane. This particular geometrical configuration is taken into account in the data post-processing phase. The receiver is operated in the first 
refraction mode with an off-axis angle of $60^{\circ}$. The focal front lens of the transmitter probe is $1200 \mathrm{~mm}$ while the focal length of the receiver is $510 \mathrm{~mm}$, leading to a maximum detectable droplet size of $327 \mu \mathrm{m}$. To distinguish quantities after and before impact, the sign of the normal velocity component $w$ is used: positive and negative signs are respectively affected for droplets before and after impact. Therefore, for the following results, subscripts $b$ and $a$ design quantities before and after impact. Otherwise, due to the high liquid flow rates injected, the impingement on the surface is characterized by a great amount of expelled droplets causing wide optical signal disturbance if the measurement volume is too close to the surface. As a consequence, all measurements undertaken in this paper are obtained at a distance of $15 \mathrm{~mm}$ above the surface, ensuring a suitable quality of the optical signal.

Both transmitter probe and PDA receiver are moved with the help of a 3-D traverse system, so that it is possible to measure at several localizations above the surface. The rror magnitude is generally difficult to determine for quantities obtained with the PDA system. In such device, the dynamic of the droplet size and velocity is fixed by the optical configuration (wavelength of both beams and focal length of lenses), the photomultiplier high voltage and the electro-optical gain. However, without changing these parameters, it is possible to estimate a relative error by performing repetitive measurements in a same position in the spray. Presently, this relative error is about $15 \%$ for both mean velocity and mean diameter.

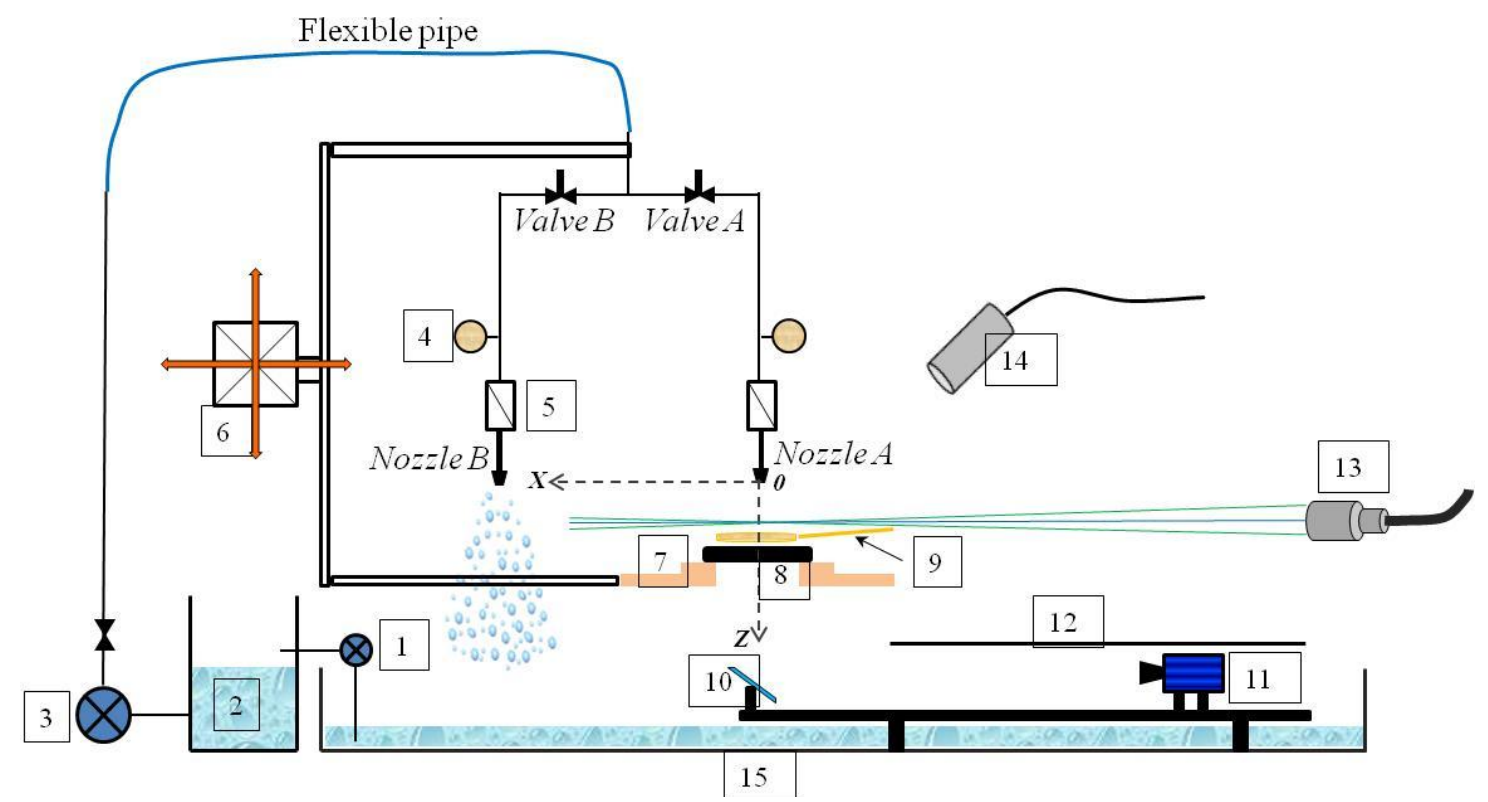

Figure 2: Experimental spray facilities and implementation of the optical measurement devices (2D-PDA and IRT). Side view (i.e. X-Z plane) of the test rig. 1 - Pump 1; 2 - Water tank; 3 Pump 2; 4 - Manometer; 5 - Flowmeter; 6 - 3D traverse system; 7 - insulation part (quartz); 8 nickel disk; 9 - retractable electromagnetic inductor; 10 - mirror; 11 - infrared camera; 12 - IR camera protective cover; 13 - laser probe; 14 - PDA receiver; 15 - main tank for water collecting.

\subsection{Heat flux density measurements}

The heat flux density removed by the spray is estimated by an inverse heat conduction method using infrared thermography (IRT) measurements [27-28]. The inverse model is based on an analytic solution of the heat equation within the nickel disk such as:

$\frac{\partial^{2} T^{\prime}}{\partial z^{2}}+\frac{\partial^{2} T^{\prime}}{\partial r^{2}}+\frac{1}{r} \frac{\partial T^{\prime}}{\partial r}+\frac{1}{r^{2}} \frac{\partial^{2} T^{\prime}}{\partial \alpha^{2}}=\frac{1}{a_{N i}} \frac{\partial T^{\prime}}{\partial t}$ 
Where $a_{N i}$ is the diffusivity of the nickel. The heat equation is associated to the following boundary and initial conditions corresponding to this relaxation experiment:

$$
\left\{\begin{array}{l}
\left.\frac{\partial T^{\prime}}{\partial r}\right|_{r=0}=0 \quad \text { and }\left.\quad \frac{\partial T^{\prime}}{\partial r}\right|_{r=R_{N i}}=0 \\
\left.k_{N i} \frac{\partial T^{\prime}}{\partial z}\right|_{z=0}=q_{F}^{\prime \prime} \quad \text { and }-\left.k_{N i} \frac{\partial T^{\prime}}{\partial z}\right|_{z=e_{N i}}=q_{R}^{\prime \prime} \\
T_{\alpha+2 \pi}^{\prime}=T_{\alpha}^{\prime} \quad T^{\prime}(r, \alpha, z, t=0)=T_{i n i t}^{\prime}(r, \alpha, z) \quad \text { and }\left.\quad \frac{\partial T^{\prime}}{\partial \alpha}\right|_{\alpha+2 \pi}=\left.\frac{\partial T^{\prime}}{\partial \alpha}\right|_{\alpha}
\end{array}\right.
$$

where $k_{N i}$ is the conductivity of the nikel, $q_{R}^{\prime \prime}$ and $q_{F}^{\prime \prime}$ are the heat fluxes $\left(W / m^{2}\right)$ corresponding respectively to all the losses on the rear face (radiation, convection and conduction through the support) and on the front face (cooling due to the sprays). Such a detailed 3D model, linking the 2D transient rear face temperature field measured by the infrared camera to the 2D removed heat flux by a single droplet on the front face, cannot be easily inverted because of the large number of parameters required. A reduced model is used here and a 2D IHC model is developed because the spray cooling is supposed to lead to symmetrical temperature fields. Such a 2D approach is possible through the introduction of an angular-averaged temperature obtained from our infrared measurements later on:

$T(r, z, t)=\frac{1}{2 \pi} \int_{0}^{2 \pi} T^{\prime}(r, \alpha, z, t) d \alpha$

$q_{\beta}(r, z, t)=\frac{1}{2 \pi} \int_{0}^{2 \pi} q_{\beta}^{\prime}(r, \alpha, z, t) d \alpha \quad$ with $\quad \beta=R, F$

Consequently the heat equation (2) becomes:

$$
\frac{\partial^{2} T}{\partial z^{2}}+\frac{\partial^{2} T}{\partial r^{2}}+\frac{1}{r} \frac{\partial T}{\partial r}=\frac{1}{a_{N i}} \frac{\partial T}{\partial t}
$$

And the associated boundary and initial conditions eqn (3) yield:

$T(r, z, t=0)=T_{\text {init }}(r, z)$

$\left.\frac{\partial T}{\partial r}\right|_{r=0}=0$ and $\left.\frac{\partial T}{\partial r}\right|_{r=R_{N i}}=0$

$\left.k_{N i} \frac{\partial T}{\partial z}\right|_{z=0}=Q^{F} \quad$ and $\quad-\left.k_{N i} \frac{\partial T}{\partial z}\right|_{z=e_{N i}}=Q^{R}$

with:

$q^{F}=q^{\prime \prime}(r, t)$ and $q^{R}=h_{E Q}^{R}\left(T_{R}-T_{\infty}\right)$

$h_{E Q}^{R}$ is the losses coefficient which gathers both convective and radiative losses, $T_{\infty}, T_{\text {stum }}, T_{F}, T_{R}$ are the surrounding, the sample holder, front and rear temperatures respectively. Eigenfunctions of the heat eqn (6) in the radial direction, with initial and boundary conditions $(7 \mathrm{a}, 7 \mathrm{~b}, 7 \mathrm{c})$, are the $J_{0}\left(\alpha_{n} r\right)$ Bessel functions, where eigenvalues $\alpha_{n}$ are solutions of Hankel (in the $r$-direction). Laplace transforms (in time) are thus performed:

$\bar{g}(r, z, p)=\int_{0}^{\infty} g(r, z, t) e^{-p t} d t \quad$ and $\quad \tilde{\bar{g}}_{n}(z, p)=\int_{0}^{R_{N i}} \bar{g}(r, z, p) r J_{0}\left(\alpha_{n} r\right) d r$

with $g=T$ or $q^{\prime \prime}$. 
Writing $\theta=T-T_{\text {init }}$, the heat equation (6) becomes a Bessel differential eqn (10) and thus an exact solution can be obtained in the transformed spaces (11):

$\frac{\partial^{2} \tilde{\bar{\theta}}_{n}}{\partial z^{2}}-\left(\alpha_{n}^{2}+\frac{p}{a_{N i}}\right) \tilde{\bar{\theta}}_{n}=0$

We assume that the initial temperature distribution is uniform after induction heating has been stopped. Solution of eqn (10) with its associated boundary conditions can be put under a matrix form using the quadrupole representation [29], for each Hankel's harmonic $n(n \geq 0)$ :

$\left[\begin{array}{c}\tilde{\bar{\theta}}_{n}^{F} \\ \tilde{\bar{q}}_{n}^{F}\end{array}\right]=\left[\begin{array}{cc}A_{n} & B_{n} \\ C_{n} & D_{n}\end{array}\right]\left[\begin{array}{l}\tilde{\bar{\theta}}_{n}^{R} \\ \tilde{\bar{q}}_{n}^{R}\end{array}\right]$

where

$A_{n}=D_{n}=\cosh \left(\gamma_{n} e_{N i}\right) ; B_{n}=\frac{1}{\lambda_{N i} \gamma_{n}} \sinh \left(\gamma_{n} e_{N i}\right)$ and $C_{n}=\lambda_{N i} \gamma_{n} \sinh \left(\gamma_{n} e_{N i}\right)$

with $\gamma_{n}^{2}=\frac{p+a_{N i} \alpha_{n}^{2}}{a_{N i}}$

Eqn (11) gives the analytical expression of the temperature profile on the rear face as a function of total heat losses (convection, radiation, and spray cooling):

$\tilde{\bar{\theta}}_{n}^{R}=-\tilde{\bar{Z}}_{n}^{A} \tilde{\bar{q}}_{n}+\tilde{\bar{Z}}_{n}^{B} \frac{\tilde{\theta}_{n, \infty}}{p}$

Two transfer functions appear in the previous solution (two impedances $\tilde{Z}_{n}^{L}$ with $\mathrm{L}=\mathrm{A}$ ). These thermal impedances depend on the solid thermal properties $(\lambda, a)$, geometric characteristics $(e)$ of the nickel disk and on the heat losses $\left(h_{E Q}^{R}\right)$. After some calculations using eqns (11), (12), boundary conditions (7) and shift property of Laplace function, it yields:

$\tilde{\bar{Z}}_{n}^{A}(p)^{-1}=h_{E Q}^{R} \cosh \left(e_{N i} \gamma_{n}\right)+\lambda_{N i} \gamma_{n} \sinh \left(e_{N i} \gamma_{n}\right)$

$\tilde{\bar{Z}}_{n}^{B}(p)=h_{E Q}^{R} \cosh \left(e_{N i} \gamma_{n}\right) \tilde{\bar{Z}}_{n}^{A}(p)$

By returning in the time domain, via a numerical Laplace inversion, two convolution products appear, noted "* *:

$\tilde{\theta}_{n}^{R}(t)=-\left[e^{-a_{N i} \alpha_{n}^{2} t} \tilde{Z}_{n}^{A}(t)\right] * \tilde{q}_{n}(t)+\left[\int_{0}^{t} e^{-a_{N i} \alpha_{n}^{2} t} \tilde{Z}_{n}^{B}\left(t^{\prime}\right) d t^{\prime}\right] \tilde{\theta}_{n, \infty}$

They can be written as:

$\left.\tilde{\theta}_{n}^{R}(t)-\left[\int_{0}^{t} e^{-a_{N i} \alpha_{n}^{2} t} \tilde{Z}_{n}^{B}\left(t^{\prime}\right) d t^{\prime}\right] \tilde{\theta}_{n, \infty}, t\right)=-\left[e^{-a_{N i} \alpha_{n}^{2} t} \tilde{Z}_{n}^{A}(t)\right] * \tilde{q}_{n}(t)$

In a matrix form, eqn (15) leads to a linear relationship between the $\mathrm{n}^{\text {th }}$ harmonic of the transformed heat flux vector $(\tilde{\mathbf{q}})$ and the $\mathrm{n}^{\text {th }}$ harmonic of the rear face temperature vector $\left(\tilde{\boldsymbol{\theta}}_{\mathrm{n}}^{\mathrm{R}}\right)$. It can be put under a matrix form:

$\tilde{\boldsymbol{\theta}}_{\mathrm{n}}^{\mathrm{R}, \mathrm{corr}}=\tilde{\mathbf{X}}_{\mathbf{n}} \widetilde{\mathbf{q}}_{\mathbf{n}}$

$\tilde{\mathbf{X}}_{\mathbf{n}}$ is a lower triangular matrix standing for the sensity matrix linking the temperature responses (for a time step $\Delta t$ ) to droplet/wall heat flux input in the Hankel domain. $\tilde{\boldsymbol{\theta}}_{\mathrm{n}}^{\mathrm{R} \text {,corr }}$ is a temperature vector which accounts for losses.

$\tilde{\boldsymbol{\theta}}_{\mathrm{n}}^{\mathrm{R}, \text { corr }}$ can be build from experimental measurement on the rear face of the nickel disk (opposite to the spray impact) provided by the infrared camera (Cedip ${ }^{\circledR}$ Jade III; spatial resolution of $320 \times 240$ pixels), 
equipped with a narrow-bandwidth spectral filter [3.97-4.01 $\mu \mathrm{m}]$. Previously, the calibration of the camera is performed by heating the disk up to $800^{\circ} \mathrm{C}$ and by recording simultaneously the infrared signal and the temperature with the use of a K-thermocouple inserted just $2 \mathrm{~mm}$ below the front face of the nickel disk, while the disk cools down naturally. Due to the limited sensibility of the camera, it should be also added that the lowest measurable by IRT is about $100^{\circ} \mathrm{C}$ corresponding roughly to a dimensionless temperature of about 0.20 . Since the cooling phase has a typical duration of about $20 \mathrm{~s}$, the selected number of frames per run is about 2000. Thus, the sampling frequency was adjusted at about $60 \mathrm{~Hz}$. Besides, both temperature $T_{w}(r, t)$ and heat flux density $q$ "' $(r, t)$ are computed at the front face $(Z=0)$. Due to the presence of the insulating holder that maintained the nickel disk, the disk area visualized by the IR camera was truncated. Thus, only a limited part of the surface can be investigated, up to $r / R=0.75$. The uncertainty resulting from the use of an inverse heat transfer algorithm cannot be directly assessed, even if the error in every data (thermophysical properties and temperature measurement) is known, another bias can be introduced if the heat conduction model is unrealistic. The estimation of the bias in the heat flux must be done using simulated temperature obtained after a direct simulation of the set of equation, Gradeck et al. [27]. The obtained temperature field is then used as input data of the inverse model in order to compare the outcomes of the inversion procedure with the primary input data (i.e. boundary conditions for direct simulations). From these tests, the uncertainties on the estimation of the fluxes and temperature are less than $10 \%$.

\section{Operating conditions and experimental procedure}

Six full cones sprays $\left(\right.$ Lechler $^{\circledR}$ nozzles) having different flow rates and cone angles were used in order to vary the liquid mass flux distribution impinging the surface and the impact conditions, i.e. sizes, velocities and trajectories of the incoming droplets before the impact. Table 1 provides the main characteristics for the six sprays. Nozzles 1-3 are those used in [26] and nozzles 4-6 correspond to new ones selected in order to keep the injection pressures and spray angles of nozzles 1-3 but an increase the liquid mass flux. For instance, nozzle 1 and 5 have the same cone angle and injection pressure but nozzle 5 can be operated with a liquid flow rate twice higher.

Table 1: Characteristics parameters of the sprays considered in the present study.

\begin{tabular}{|c|c|c|c|c|c|}
\hline $\begin{array}{c}\text { Nozzle and spray } \\
\text { number }\end{array}$ & $\begin{array}{c}\text { Lechler } \\
\text { reference }\end{array}$ & $\begin{array}{c}\text { Spray angle } \\
\left({ }^{\circ}\right)\end{array}$ & $\begin{array}{c}\text { Flow rate } \\
(\mathbf{l} / \mathbf{m i n})\end{array}$ & $\begin{array}{c}\text { Injection } \\
\text { pressure } \\
(\mathbf{b a r})\end{array}$ & $\begin{array}{c}\text { Nozzle-disk } \\
\text { distance } \\
(\mathbf{m m})\end{array}$ \\
\hline 1 & 460.643 .17 & 45 & 6.7 & 7 & 250 \\
\hline 2 & 460.683 .17 & 45 & 6.7 & 3.7 & 250 \\
\hline 3 & 460.684 .17 & 60 & 6.7 & 3.7 & 160 \\
\hline 4 & 460.724 .17 & 60 & 9 & 3.7 & 160 \\
\hline 5 & 460.783 .17 & 45 & 13.4 & 7 & 250 \\
\hline 6 & 460.804 .17 & 60 & 13.4 & 3.7 & 160 \\
\hline
\end{tabular}

The vertical distance between the exit of each of the nozzles and the surface is adapted according the spray angle in order to ensure that the spray covers roughly the entire disk surface. The liquid mass flux distribution $G$ on the disk surface disk is measured for each spray with a patternator (composed of a row of twelve round tubes) in absence of the disk so that the top of the tubes are located at the same $Z$ elevation as the front face of the disk. A full description of this device can be found in [26]. In order to match easily the liquid mass flux with the heat flux extracted by the liquid, an angular averaging of liquid mass flux measurements is also performed. As a result, the liquid mass flux distributions along the radial position $r$ for the six sprays are presented in Figure 3. It seems that according the spray angle value, the liquid mass flux distribution differs. Nozzles with angles of $60^{\circ}$ (i.e. nozzles 3,4 and 6) lead to a distribution that decreases monotonously from the center up the edge of the disk. For spray angle 
of $45^{\circ}$ (i.e. nozzles 1,2 and 5), the distributions describe first a plateau and then, experience a maximum at a given radial position before decreasing toward the edge.

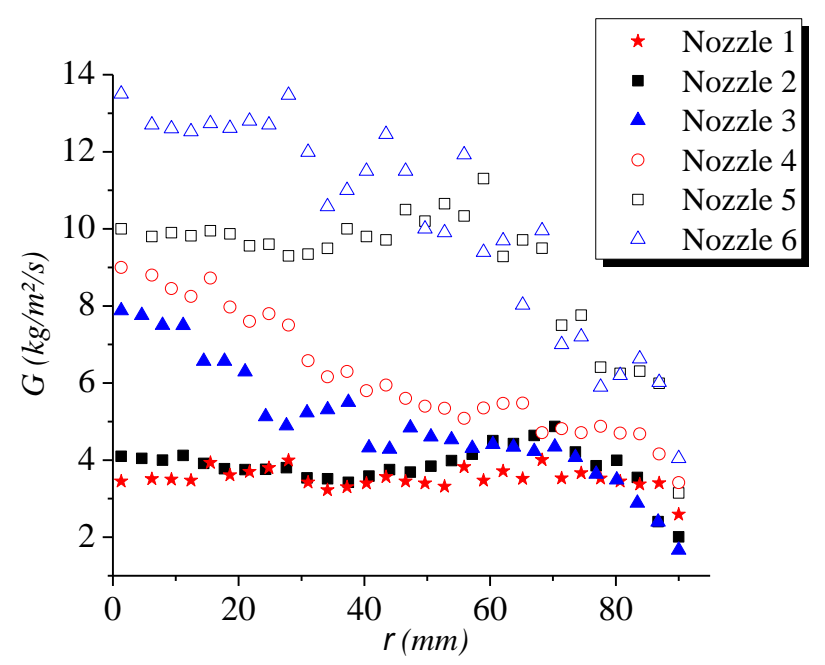

Figure 3. Liquid mass flux distributions of the six sprays along the radial position $r$ of the disk.

As mentioned previously, each experiment begins by heating the disk up to a surface temperature of about $800^{\circ} \mathrm{C}$ (controlled by the IR camera). Then, the cooling process is started and both IRT and PDA techniques are synchronized to follow the cooling phase. The trigger output of the PDA processor is used to drive the IR camera and the spray operations (switching of both electro-valves). This procedure allows to monitor simultaneously the history of the heat flux as well as the droplets properties before and after impact. In the following, the temperature of the liquid at the injection is the ambient temperature (i.e. about) $20^{\circ} \mathrm{C}$

Because PDA technique is a pointwise measurement, three synchronized IRT/PDA measurements are repeated for each spray in order to investigate the incoming and outgoing spray properties at $r / R=0$, 0.5 and 0.75 , and for a distance of $15 \mathrm{~mm}$ from the surface. As a result, a set of 18 tests is performed, as shown in Table 2. Besides, in order to verify the repeatability of the cooling for a given test, each measurement is repeated twice. In fact, the experiments show that the non-repeatability concerns mainly the Leidenfrost temperature which can be different between the two consecutive measurements at a same position.

Table 2. Description of the conducted experiments.

\begin{tabular}{|c|c|cl|}
\hline $\boldsymbol{r} / \boldsymbol{R}$ & Sprays & Test number & \\
\hline 0 & 1 to 6 & 1 to 6 & (two measurements for each spray) \\
\hline 0.5 & 1 to 6 & 7 to 12 & (two measurements for each spray) \\
\hline 0.75 & 1 to 6 & 13 to 18 & (two measurements for each spray) \\
\hline
\end{tabular}

Table 3 summarizes the range of main measured quantities for the six sprays describing the impingement conditions for the present study. As it will be discussed in section 6.2, the presence of the disk has a significant effect on the size distributions and the trajectories of the incoming droplets. Therefore, these impingement conditions have to be measured with the disk. This operation is possible with the PDA system whereas it is not the case for liquid mass flux distribution. As a result, both mean arithmetic diameter $D_{10 b}$ and velocity $\bar{w}_{b}$ in Table 3 are obtained from PDA measurements in the presence of the disk (not heated) $15 \mathrm{~mm}$ above the surface for the three $r / R$ positions. Then, a mean normal Weber number before impact $\overline{W e}_{n b}$ can be deduced as follow:

$\overline{W e}_{n b}=\frac{\rho \bar{w}_{b}^{2} D_{10 b}}{\sigma}$ 
Table 3: Summary of the range of variation of the parameters characterizing the impingement conditions in the case of the presence of a non-heated disk.

\begin{tabular}{|c|c|c|c|c|c|c|}
\hline Sprays & $\begin{array}{c}\text { Liquid } \\
\text { mass flux } \\
\boldsymbol{G} \\
\left(\mathrm{kg} / \mathrm{m}^{2} / \mathrm{s}\right)\end{array}$ & $\begin{array}{c}\text { Mean diam- } \\
\text { eter before } \\
\text { impact } \boldsymbol{D}_{\mathbf{1 0 b}} \\
(\mu \mathrm{m})\end{array}$ & $\begin{array}{c}\text { Mean } \\
\text { normal } \\
\text { velocity } \\
\bar{w}_{b}(\mathbf{m} / \mathbf{s})\end{array}$ & $\begin{array}{c}\text { Sauter Di- } \\
\text { ameter be- } \\
\text { fore impact } \\
\boldsymbol{D}_{\mathbf{3 2 b} \boldsymbol{b}}(\mu \mathrm{m})\end{array}$ & $\begin{array}{c}\text { Normal We- } \\
\text { ber before } \\
\text { impact } \\
\overline{W e}_{n b}(-)\end{array}$ & $\begin{array}{c}\text { Impact an- } \\
\text { gle } \boldsymbol{\alpha}\left(^{\circ}\right)\end{array}$ \\
\hline $\begin{array}{c}\text { Full } \\
\text { cones }\end{array}$ & $1.7-13.5$ & $71.8-91.5$ & $7.4-14.8$ & $135-197$ & $49-330$ & $0-30^{\circ}$ \\
\hline
\end{tabular}

In Table 3, the liquid mass flux is directly deduced from Figure 3. The range of variation of the normal Weber number is significant (about a factor 30), while the mean diameter varies in a more limited range. Its variation is then correlated of the vertical velocity $\bar{w}_{b}$. Moreover, the measurements show that the normal Weber number decreases toward the edge of the disk.

\section{Heat flux results}

In this section, main results that concerns heat flux extracted at the disk surface are presented. Figure 4 depicts typical boiling curves (i.e. heat flux $q$ " plotted as a function of the wall temperature $\left.T_{w}\right)$ obtained with the six sprays at the center of the disk $(r / R=0)$. The curves display quite well the three main regimes:

$i$ - $\quad$ The Leidenfrost regime (or film boiling regime) occurs first for the highest wall temperatures where the extracted heat flux are moderate,

ii- $\quad$ Then, by decreasing the wall temperature, the transient boiling regime appears and is characterized by the partial deposition of droplets on the surface and the formation of a liquid film. Some vapour bubbles appear in the film, leading to an increase of the heat transfer up to a maximum of the heat flux, called critical heat flux (CHF).

iii- In the final phase, beyond the CHF, when the wall temperature decreases again, the nucleate boiling regime appears: the heat flux decreases due to a sharp decrease of the number of vapour bubbles.

In addition, as expected: both the extracted heat flux and the Leidenfrost temperature increase with the liquid mass flux $G$.

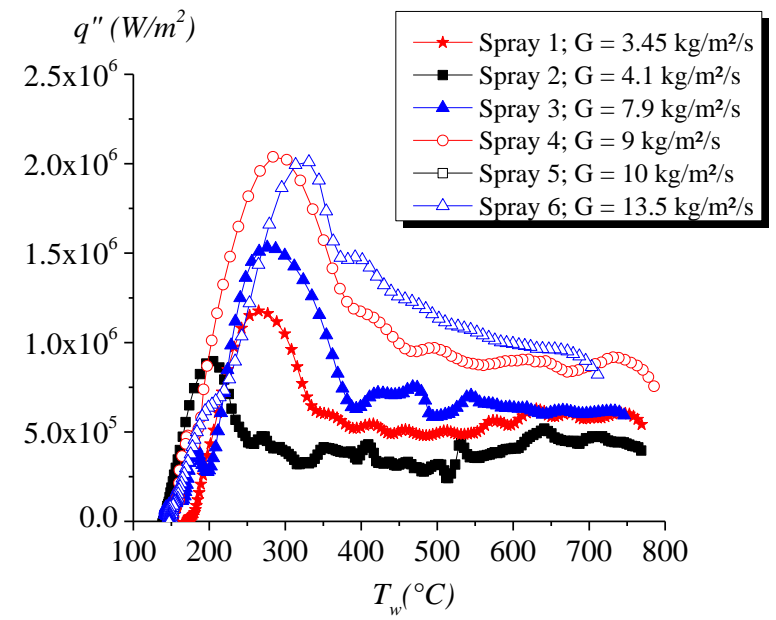

Figure 4: Boiling curves for the six sprays plotted at $r / R=0$.

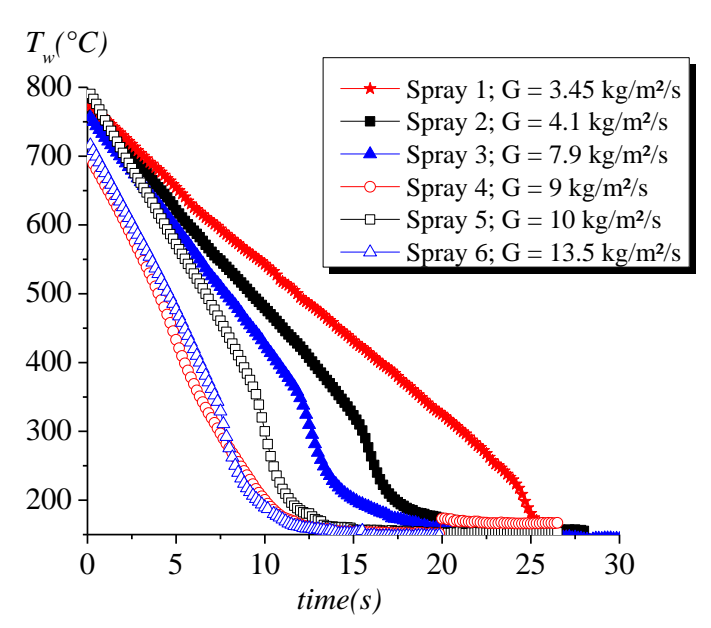

Figure 5: Evolution of the wall temperature as a function of the time for the six sprays plotted at $r / R=0$. 
For the following investigation of the hydrodynamic impingement, it is essential to know the Leidenfrost temperature corresponding to the minimal temperature for which the vapour layer appears. This temperature can be determined by locating the point where the boiling curves experience a minimum. However, for high liquid mass fluxes, it is more difficult to identify this point. Alternatively, the Leidenfrost temperature can be identified from the surface temperature versus time. This happens when the negative slope of the curve suddenly becomes steeper. Therefore, this alternative method was preferred in the present work . In section 3., it was mentioned that a problem of nonrepeatability for the Leidenfrost temperature value can occur. In this case, the mean value of both Leidenfrost temperature values deduced from the two consecutive experiments is used. An example is given in Figure 5 for the six sprays at $r / R=0$. Moreover, as expected, the duration of the cooling phase is reduced by increasing the liquid mass flux. The work of Yao \& Cox [30] showed that $T_{\text {Leid }}$ can be linked to a specific spray Weber number according to the following correlation:

$T_{\text {leid }}=1400 W e_{S}^{0.13}$

where the spray Weber number is based on the Sauter Mean Diameter $D_{32}$ (SMD) and on the liquid mass flux $G$ :

$W e_{s}=\frac{G^{2} D_{32}}{\rho \sigma}$

The Leidenfrost temperatures for the 18 tests as a function of the spray Weber number are reported in Figure 6. In the previous study [26], the spray Weber number reaches a maximum value of about $2.10^{-4}$ and in the present study, where the liquid mass flux is increased, the maximum value is doubled. The correlation of Yao \& Cox is also reported in Figure 6: it appears that $T_{\text {Leid }}$ increases with $W e_{s}$. Usually, the cooling ability of a spray can be quantified by the cooling efficiency $\xi$, defined by [31]:

$$
\xi=\frac{q^{\prime \prime}}{G\left[h_{v}+c_{p l}\left(T_{s a t}-T_{l}\right)+c_{p v}\left(T_{w}-T_{s a t}\right)\right]}
$$

It represents the amount of heat extracted by the spray divided by the total heat transfer which is required for complete evaporation of the liquid, including the power required to heat the liquid up to its saturation temperature and to heat the vapor up to the wall temperature. Yao \& Cox [30] proposed an empirical correlation of the efficiency based on the spray Weber number and the wall temperature $T_{w}$ :

$\zeta=8 \times 10^{-7}\left[\frac{W e_{s} T_{s a t}}{T_{w}-T_{l}}\right]^{-0.62}+3.5 \times 10^{-3}\left[\frac{W e_{s} T_{s a t}}{T_{w}-T_{l}}\right]^{-0.2}$

This correlation was obtained for wall temperatures varying from $700^{\circ} \mathrm{C}$ up to $300^{\circ} \mathrm{C}$, i.e. only for the Leidenfrost regime. In the current work, knowing the distribution of the liquid mass flux, the SMD and the wall temperature, the quantity $W e_{s} T_{s a t} /\left(T_{w}-T_{l}\right)$ can be easily calculated. Therefore, it is possible to compare the cooling efficiency determined in the present study (eqn. (21)) with the correlation of Yao \& Cox (eqn 22). Figure 7 depicts the evolution of the cooling efficiency as a function of $W e_{s} \cdot T_{s a t} /\left(T_{w}-T_{s a t}\right)$ obtained with the 18 sets of experiments for temperatures above the Leidenfrost one (from $715^{\circ} \mathrm{C}$ up to $500^{\circ} \mathrm{C}$ ). It means that for a given wall temperature, only the $W e_{s}$ varies. The correlation of Yao \& Cox (eqn (22)) is also plotted in parallel. The trend obtained with the present experimental data is similar to the one obtained by Yao \& Cox. Using the method of Yao \& Cox based on the Weber spray and the wall temperature, a new correlation obtained from the current experimental data may be proposed:

$\zeta=2.6 \times 10^{-3}\left[\frac{W e_{s} T_{\text {sat }}}{T_{w}-T_{l}}\right]^{-0.227}$

Although only tone erm is found, this new correlation is very close to those of Yao \& Cox. 


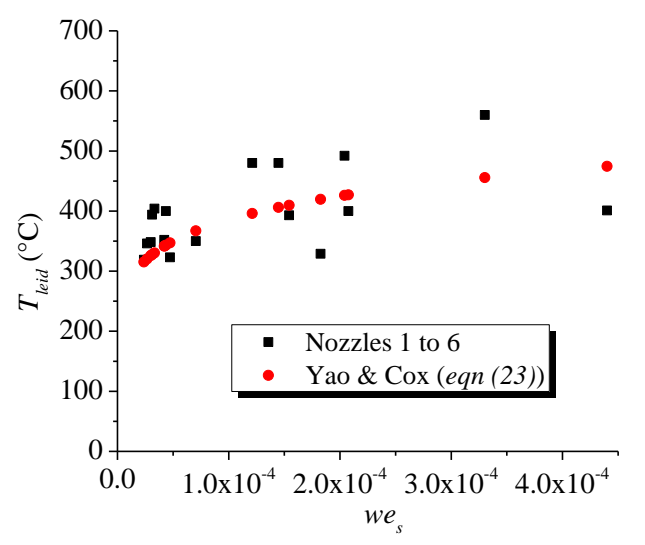

Figure 6: Evolution of the Leidenfrost temperature as a function of the spray Weber number for the 18 sets and comparison with the correlation of Yao\&Cox [30].

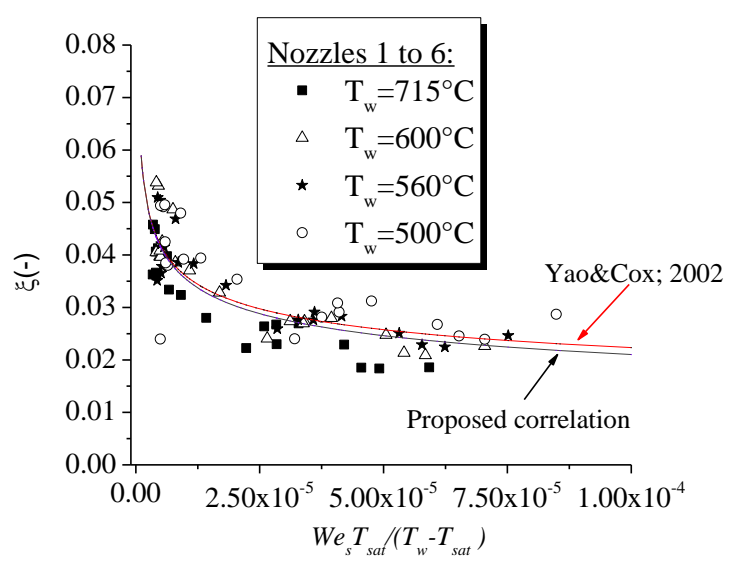

Figure 7: Comparison of the cooling efficiency obtained in the present study for several wall temperatures with the correlation of Yao\&Cox [30].

\section{Data reduction procedure for the Phase Doppler measurements during the cooling phase}

\subsection{Principle of the procedure}

This section is devoted to the post-processing procedure of the experimental data that allows the analysis of the evolution of the spray properties as a function of the surface temperature during the cooling phase. In particular, the processing is based on the previous heat flux results. The principle of this method is illustrated in Figure 8 by taking the example of the spray 1. The upper part shows the evolution of the heat flux and the dimensionless wall temperature $T^{*}$ as a function of the time, i.e. during the cooling. The lower part represents the time interval during which the PDA data were recorded simultaneously. As both techniques are synchronized, PDA and IRT data were analysed for $n$ time intervals of duration $\delta t$, the value of $n$ being chosen by the user. For each interval $\delta t$, the droplet diameter and velocity distributions were obtained from PDA measurements and a time averaged wall temperature was derived.

\subsection{Discussion and validation of the procedure}

A key issue of this procedure is the number of required samples at each $r / R$ position to ensure reliable value of the computed arithmetic mean quantities. The number of required droplets at each interval $\delta t$ can be estimated by using the following expression:

$\varepsilon^{2}=\frac{1}{N} \frac{\sigma_{\varphi}^{2}}{\bar{\varphi}^{2}}$

where $\varepsilon$ is the error level on the mean value of $\varphi$ due to statistical convergence, $N$ the number of samples, $\sigma_{\varphi}^{2}$ and $\bar{\varphi}$ the variance and the arithmetic mean of the quantity $\varphi$ itself respectively. To analyze the anticipated potential error due, the worst case is considered. For that purpose, the measurement performed with the spray 1 at $r / R=0$ with a total number of 25130 droplets and a number of $n=10$ intervals $\delta t$ is chosen. The error level is calculated for the mean diameter before impact $D_{10 b}$ at each time interval $\delta t$. The values of the mean diameter and its variance are given by using the sampling procedure. The results are displayed in Table 4, which reveal that the statistical convergence error is estimated with a maximum of $2.2 \%$.

Another fundamental point is the number of interval time that one can choose to analyse properly the spray impingement. The mean result $D_{10 b}$ versus the time for several numbers $n$ of interval time in the case of the spray (1) at $r / R=0$ is plotted in Figure 9. Two main comments can be deduced: 
i- $\quad$ The curve describing the evolution of $D_{10 b}$ does not depend on the number $n$.

ii- $\quad$ The overall evolution of $D_{10 b}$ can be divided in two phases. A first one from $t=0$ up to about $12 \mathrm{~s}$ where $D_{10 b}$ seems to be constant. And a second phase from $t \approx 12 \mathrm{~s}$ up to the end of the cooling during where $D_{10 b}$ increases continuously. On the other hand, according to Figure 8 , it seems that the time $t \approx 12 \mathrm{~s}$ corresponds roughly to the Leidenfrost point.
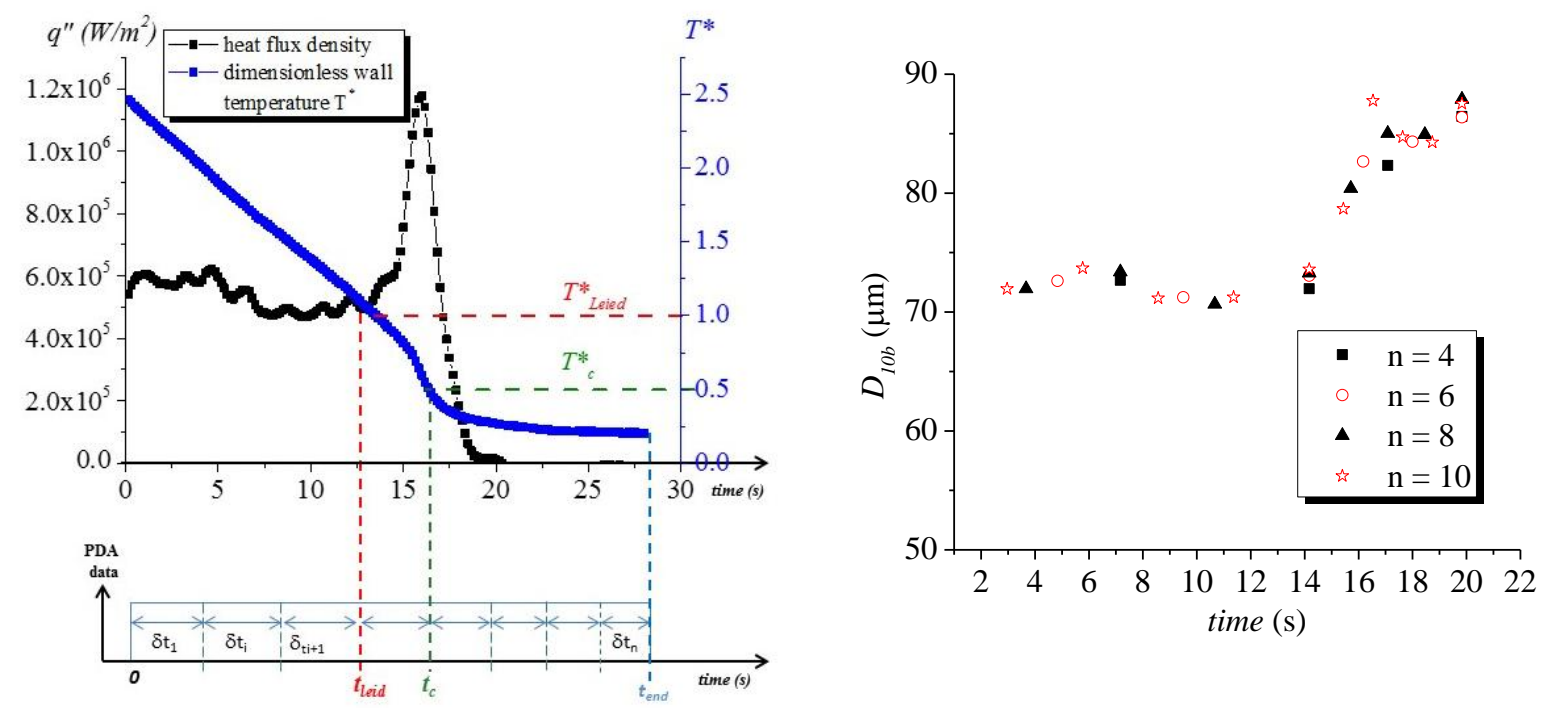

Figure 8: Typical example of the time evolution of the extracted heat flux and wall temperature. Sampling procedure for the phase Doppler measurements analysis.

Figure 9: Evolution of the mean droplet diameter before impact for several time interval $n$ (Spray (1) at $r / R=0$ ).

Table 4: Estimation of the error level for the mean diameter before impact during a cooling experiment for different successive time steps.

\begin{tabular}{|c|c|c|c|c|c|}
\hline number of $\boldsymbol{\delta} \boldsymbol{t}$ & time (s) & $\begin{array}{c}\text { Variance } \sigma_{\varphi}^{2} \\
\text { of } \boldsymbol{D}\end{array}$ & $\boldsymbol{D}_{\mathbf{1 0 b}}(\boldsymbol{\mu m})$ & $\mathbf{N}$ & $\boldsymbol{\varepsilon}(\boldsymbol{\%})$ \\
\hline 1 & 3.0 & 1914 & 71.95 & 3424 & 1.0 \\
2 & 5.8 & 1958 & 73.69 & 3317 & 1.0 \\
3 & 8.6 & 1533 & 71.16 & 3195 & 1.0 \\
4 & 11.4 & 1689 & 71.25 & 3374 & 1.0 \\
5 & 14.2 & 1787 & 73.60 & 3186 & 1.0 \\
6 & 15.4 & 1832 & 78.68 & 731 & 2.0 \\
7 & 16.5 & 2091 & 87.77 & 588 & 2.1 \\
8 & 17.6 & 1773 & 84.72 & 494 & 2.2 \\
9 & 18.7 & 2003 & 84.28 & 625 & 2.1 \\
10 & 19.8 & 2167 & 87.53 & 633 & 2.1 \\
\hline
\end{tabular}

Therefore, although the temperature decreases continuously, two phases are proposed: a first steady phase corresponding to the Leidenfrost regime where no variation is observed and a second one, for surface temperatures lower than the Leidenfrost one, when strong variations occur. As a result, 
three time intervals $\delta t(n=3)$, or dimensionless temperatures intervals, are chosen to analyse the impingement during the cooling:

(1) $0<t<t_{\text {Leid }}$ corresponding to the Leidenfrost regime $\left(T^{*}>1\right)$.

(2) $t_{\text {Leid }}<t<t_{c}$ where $t_{c}$ is the time when $T^{*}=0.5$ or when $T_{w}=T_{\text {sat }}$ (corresponding roughly to the CHF point). During this interval time, the dimensionless temperature interval is $0.5<T^{*}<1$.

$t_{c}<t$ corresponding to the nucleate boiling regime $\left(0.2<T^{*}<0.5\right)$. As mentioned before in section 2., no temperature below $100^{\circ} \mathrm{C}$ are available.

\section{Refined study of the hydrodynamic conditions of the spray impingement}

\subsection{Mapping of the impingement regimes}

A first application of the previous procedure is to generalize the boundaries given in Figure 1 (for a single droplet) for sprays. For that purpose, the six sprays and each investigated $r / R$ positions were considered and the three time intervals previously defined are employed. Then, for a given test (i.e. for a given spray and a given $r / R$ position) and for one interval $\delta t$, the Mundo number $K$ and the dimensionless temperature $T^{*}$ are calculated according to the following steps:

$i$. A time average dimensionless temperature $\tilde{T}^{*}$ is derived, using IRT measurements.

ii. A time average mean diameter $\widetilde{D}_{10 b}$ and a time averaged mean normal velocity $\widetilde{w}_{b}$ before impact are determined using PDA measurements

iii. $\quad$ A time average normal Weber $\widetilde{W} e_{n b}$ can be deduced using eqn (2) as well as a time average Ohnesorge number defined by:

$$
\widetilde{O h}=\frac{\mu}{\rho \widetilde{D}_{10 b} \sigma}
$$

iv. $\quad$ Finally, a time average Mundo number can be deduced by:

$$
\widetilde{K}=\widetilde{W} e_{n b} \widetilde{O h}
$$

$v$. Steps $i$. to $i v$. are repeated for the two others intervals and the other experiment sets.

Finally, a map of the different regimes is obtained in the diagram $\left(T^{*}, \log (K)\right)$ (Figure 10), where the results for impacts onto a surface at room temperature are also added (cold impact: blue stars, for $\left.T^{*}<0\right)$.

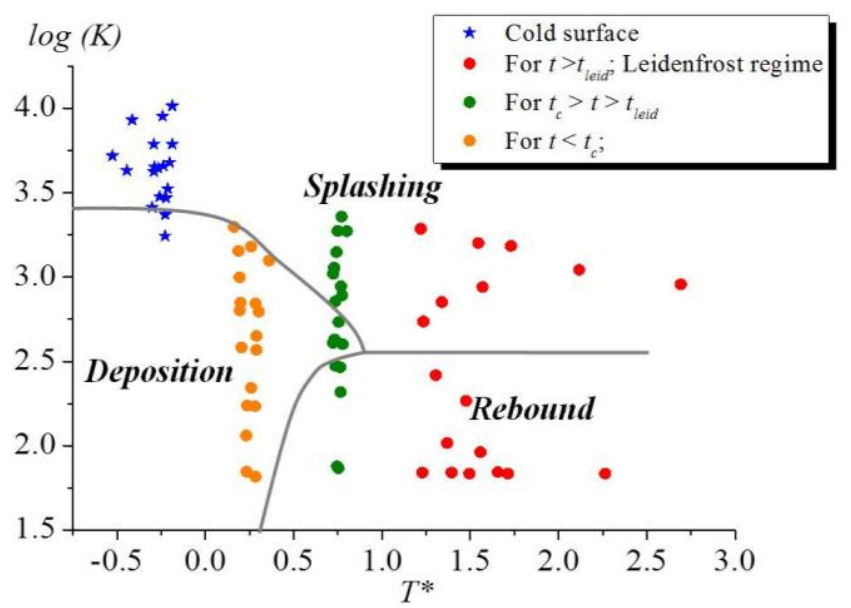

Figure 10: Mapping of the main impingement regimes obtained with the measurements obtained for the different sprays. 
For $T^{*}>1$, both rebound and splashing regimes can be observed, according to the Mundo number value. When $0.5<T^{*}<1$, the three regimes are obtained. On contrary, only deposition seems to occur for $0.20<T^{*}<0.5$, meaning that a liquid film appears along the surface. In the case of the cold impact, the splashing regime is mainly observed. The results obtained for heat transfer will be interpreted in the light of the different observed regimes.

\subsection{Effect of the wall on the incoming spray properties}

A brief literature review shows that the incoming droplet properties of a spray are generally measured in absence of any solid wall, even if the goal is to characterize the impingements of the droplets on a solid surface. However, the presence of the surface is likely to influence the trajectories of the droplets, by modifying the entrained airflow, and therefore both their impingement characteristics and the corresponding heat transfers. This phenomenon was already studied in [19-21] but only for a "cold impingement". In this section, a heated surface is considered. In particular, the properties of droplet before impact during the cooling phase are compared with those in absence of the solid wall (free spray). To illustrate this point, results obtained with only one spray among the six ones are displayed, but all the conclusions may be extended for the other sprays. Figure 11 presents the droplet size distribution for the spray (1) and in the case of a cooling experiment at $r / R=0$ (Figure 11) and at $r / R=0.75$ (Figure 11b).

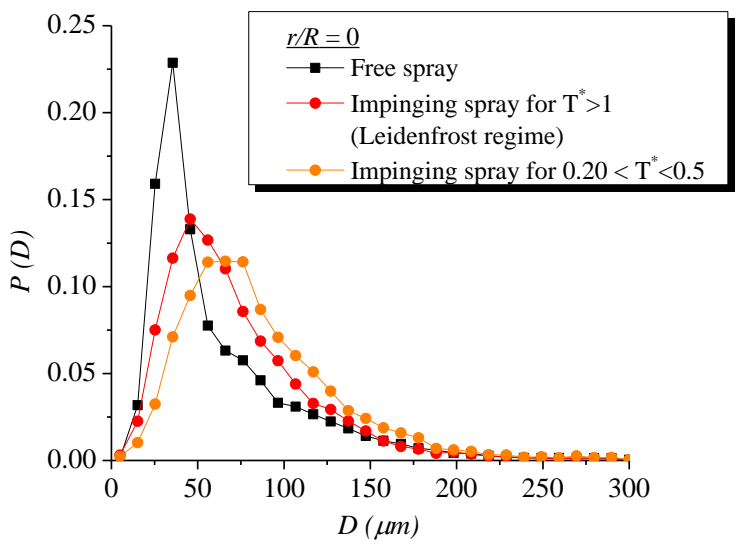

(a)

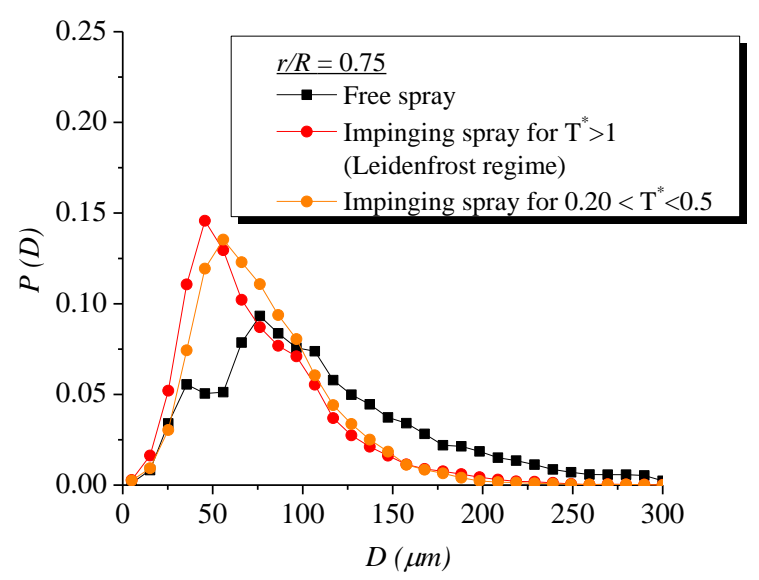

(b)

Figure 11: Comparison of the size distributions of the droplets before impact in the case of a free spray and in presence of the disk, for the spray (1) measured at $X / R=0$ (a) and at $X / R=0.75 \mathrm{~mm}(\mathrm{~b})$.

In the case of the cooling experiments, the droplet size distributions are time averaged distributions calculated over two time intervals corresponding to $T^{*}>1$ and $0.20<T^{*}<0.5$ (i.e. during the Leidenfrost and nucleate boiling regime respectively). At $r / R=0$ (Figure 11a), both distributions recorded during the cooling phase exhibit a higher population of large droplets compared to the case of the free spray. Furthermore, this effect was also observed for a "cold impingement" [26]. It was assumed that the smallest droplets (i.e. with the smallest Stokes number) were deviated by the entrained airflow towards the disk edges. At $r / R=0.75$ (Figure 11b), a larger population of smaller droplets can be observed in comparison to the case of the free spray. It could be attributed to the deviation of the smallest droplets from the central axis of the spray $(r / R=0)$ leading to an increase of the number of small droplets detected at $r / R=0.75$. In addition, it seems that the temperature has a noticeable effect: the small droplets are more deviated when the temperature decreases.

The effect of the wall temperature on the droplet deviation can be related to the occurrence of a strong vapour flow coming from the solid heated surface after the complete vanishing of the vapour film formed on the solid surface. Indeed, the droplets may directly impact on the hot surface which 
may improve strongly the vaporisation of the liquid. This strong vapour flow going mainly in the opposite direction of the spray flow, tends to deviate primarily the smallest droplets (see the sketch in Figure 12). As a result, the effect of this deviation is the increase of the mean droplet diameter when the temperature decreases observed in Figure 9.

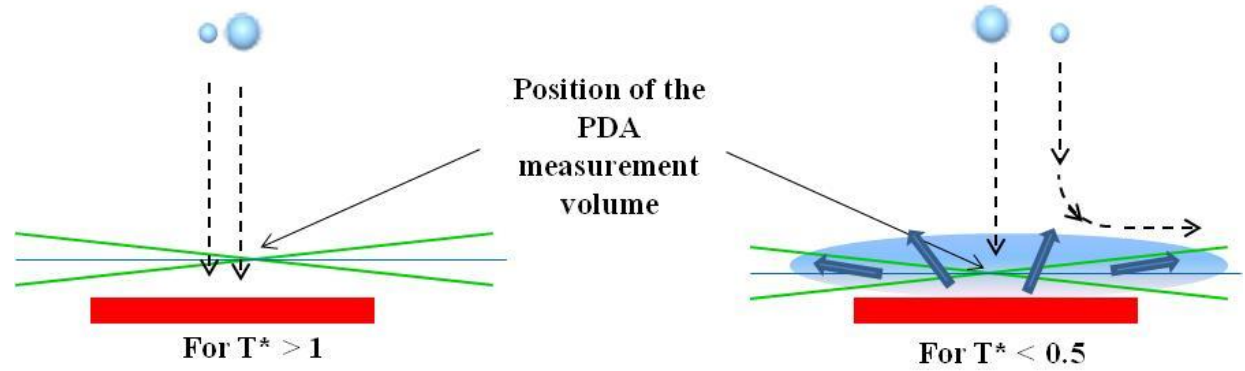

Figure 12: sketch illustrating the effect of the wall temperature on the deviation of the small droplets.

The comparison of the correlations size/velocity for the normal component (Figure 13) and the horizontal component (Figure 14) during the cooling sequence with the case of a free spray could also allow to check the combined effects of the presence of the wall and its temperature.

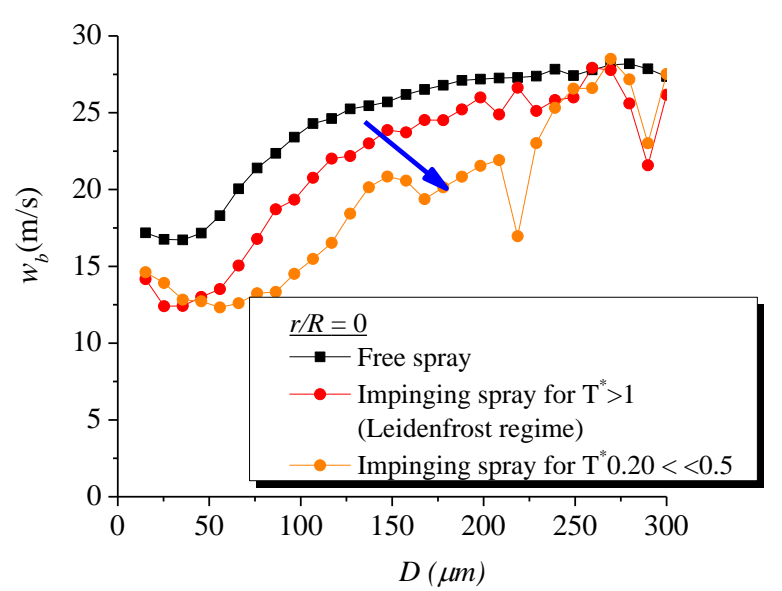

(a)

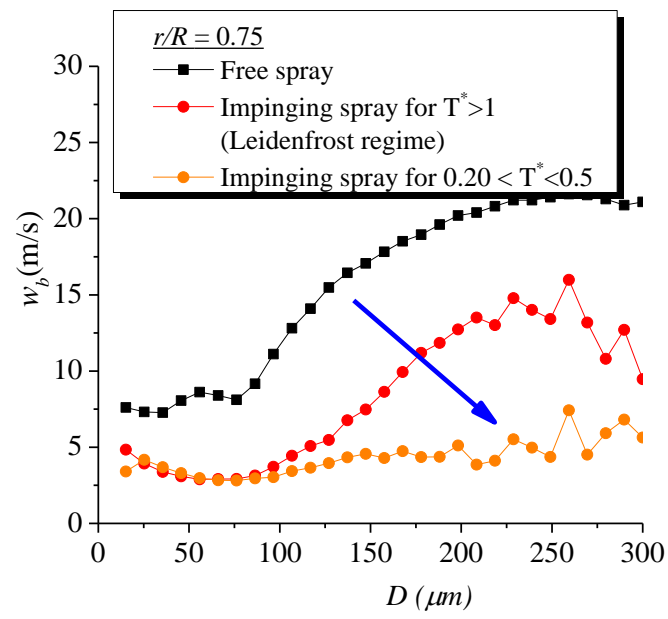

(b)

Figure 13: Correlation between droplet size and vertical velocity of the incoming droplets at $r / R=0$ (a) and at $r / R=0.75$ (b) in the case of a free spray and in presence of the disk for the spray (1) at two dimensionless temperatures.

In Figure 13, the correlations for the vertical velocity plotted at $r / R=0$ and $r / R=0.75$ show clearly that the droplets are decelerated in the presence of the disk and that this velocity reduction is more pronounced when the wall temperature decreases. In contrast, the correlation for the horizontal velocity shows a significant increase of the velocity due to the presence of the disk (Figure 14). This increase of the horizontal velocity affects mainly the smallest droplets and is more important in the vicinity of the disk edges (Figure 14b). Moreover, it can be observed also that this effect is more pronounced when the wall temperature is decreased. 


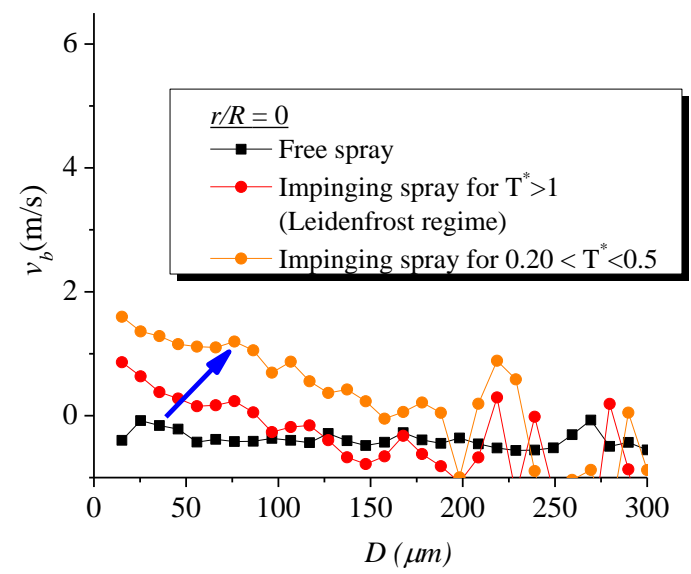

(a)

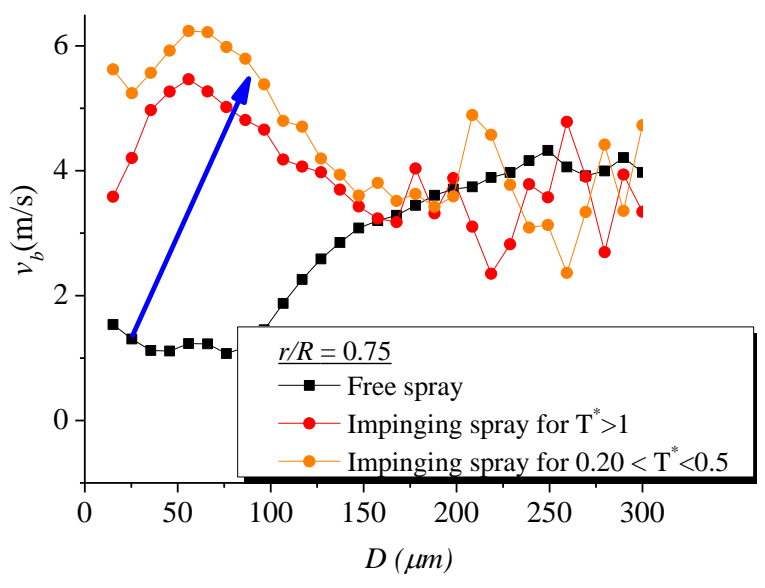

(b)

Figure 14: Correlation of the droplet size and horizontal velocity of the incoming droplets at $r / R=0$ (a) and at $r / R=0.75$ (b) in the case of a free spray and in presence of the disk for the spray (1) at two dimensionless temperatures.

\subsection{Secondary spray properties - Deep investigation of a given case}

In this section, the secondary spray properties (after impact) are investigated for one case only: spray (1) at $r / R=0$. The impingement on the surface induces droplet deformation and also induces some oscillations during the droplet flight before reaching again a spherical shape. These deformations and oscillations are more pronounced for larger droplets. As the PDA system is based on a sphericity assumption, a bias in the diameter derivation may occur. However, all measurements are performed at $15 \mathrm{~mm}$ above the surface, and mainly small droplets are considered, so one can assume that all detected droplets are spherical. A typical one single-point measurement of droplets diameter count for droplets after impact is presented in Figure 15 for the Leidenfrost and nucleate boiling regimes. It appears that the number and the size of the expelled droplets are higher during nucleate boiling regimes. To provide a potential explanation of this observation, the evolution of the mean diameter as well as the number of droplets during the cooling is analysed.

The evolution of the mean diameter before and after the impact as a function of the dimensionless temperature is given in Figure 16, using the procedure described in section 5 with $n=10$ time intervals. First, the time average mean diameter before impact $\widetilde{D}_{10 b}$ depicts the same behaviour seen in Figure 9: $\widetilde{D}_{10 b}$ does not vary much up to $T^{*} \approx 1$ and then shows a monotonous increase up to $T^{*} \approx 0.2$. The mean diameter after the impact $\widetilde{D}_{10 a}$ has a similar trend. As a result, the time average droplet ratio $\tilde{\lambda}_{D 10}$ (defined by $\widetilde{D}_{10 a} / \widetilde{D}_{10 b}$ ) follows the same behaviour. Similarly, the time average number of droplet $\widetilde{N}_{b}$ before impact and $\widetilde{N}_{a}$ after impact, as well as the time average number ratio $\tilde{\lambda}_{N}$ (defined by $\widetilde{N}_{a} / \widetilde{N}_{b}$ ) during the cooling phase is illustrated in Figure 17. While no significant change of the droplet number before impact can be noticed, the expelled droplet number seems to increase with a net increase after $T^{*} \approx 0.4$. 


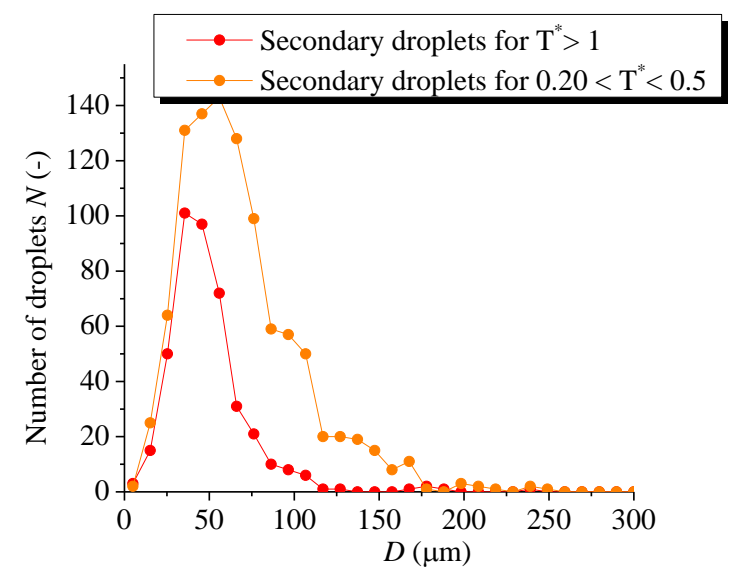

Figure 15: Droplets diameter count of expelled droplets for the spray (1) at $r / R=0$ and for two wall dimensionless temperatures.

Nevertheless, the interpretation of the increase of both $\tilde{\lambda}_{D 10}$ and $\tilde{\lambda}_{N}$ ratios remains difficult to explain. Therefore, it would be interesting to correlate the characteristics of the secondary droplets with the heat flux extracted by the fluid. Figure 18 shows the simultaneous evolution of the extracted heat flux density as a function of the dimensionless temperature, in parallel to the three time average ratios: $\tilde{\lambda}_{D 10}, \tilde{\lambda}_{N}$ and $\tilde{\lambda}_{m}$. The latter ratio is the expelled mass ratio defined as $\tilde{\lambda}_{m}=$ $\dot{\tilde{m}}_{a} / \dot{\tilde{m}}_{b}$ ) and computed as follows:

$$
\tilde{\lambda}_{m}=\frac{\sum_{i=1}^{N_{a}}\left(D_{a}^{3}\right)}{\sum_{i=1}^{N_{b}}\left(D_{b}^{3}\right)}
$$

This estimate is more accurate than when computed directly with the absolute mass flux [21]. The discussion of Figure 18 is divided in four steps according to the dimensionless temperature evolution:

i- $\quad$ For $T^{*}>1$ (Leidenfrost regime), the three ratios $\left(\tilde{\lambda}_{D 10}, \tilde{\lambda}_{N}\right.$ and $\left.\tilde{\lambda}_{m}\right)$ display a roughly constant trend. It is in good agreement with the previous statement (section 5.2) specifying a steady state of the droplet impingement whatever the surface temperature.

ii- $\quad$ For $0.4<T^{*}<1$, the three ratios increase but $\tilde{\lambda}_{N}$ increases slightly more. According to Figure 10 for these temperatures, deposition on the solid surface are potentially observed, meaning that a liquid film began to growth. The presence of the liquid film strongly influences the secondary droplets number. Indeed, some droplets are expelled from the film due to explosions of vapour bubbles within the film, but also resulting from the splashing of incoming droplets on the film. Consequently, it can explain the increase of both $\tilde{\lambda}_{N}$ and $\tilde{\lambda}_{m}$. The increase of $\tilde{\lambda}_{D 10}$, attributed to the increase of $D_{10 a}$, shows that the liquid film expelled droplets with larger droplets than during the Leidenfrost regime.

iii- When the surface temperature becomes too low to maintain a vapour layer $\left(0.25<T^{*}<0.4\right)$, the liquid film covers the entire surface of the disk. Thus, the number of expelled droplets is increased in line with the continuous increase of the expelled mass ratio.

iv- $\quad$ When the surface temperature continues to decrease (for $T^{*}<0.25$ ), the liquid film depth also continues to increase. According to Cossali et al. [2], the number of expelled droplets decreases with the increase of the depth of the film, which can be related to the slight decrease presently observed for the expelled mass ratio (Figure 18). Since the temperature measurements below $100^{\circ} \mathrm{C}$ are not possible (basically for $T^{*}<0.2$ ), it remains difficult to conclude properly about the evolution of $\tilde{\lambda}_{D 10}$ and $\tilde{\lambda}_{m}$ for $T^{*}<0.2$. 
Nevertheless, a similar evolution for the expelled mass ratio was already observed by Jia and Qiu [23] and these authors have noticed a clear decrease of the expelled mass ratio for $T^{*}<0.2$.

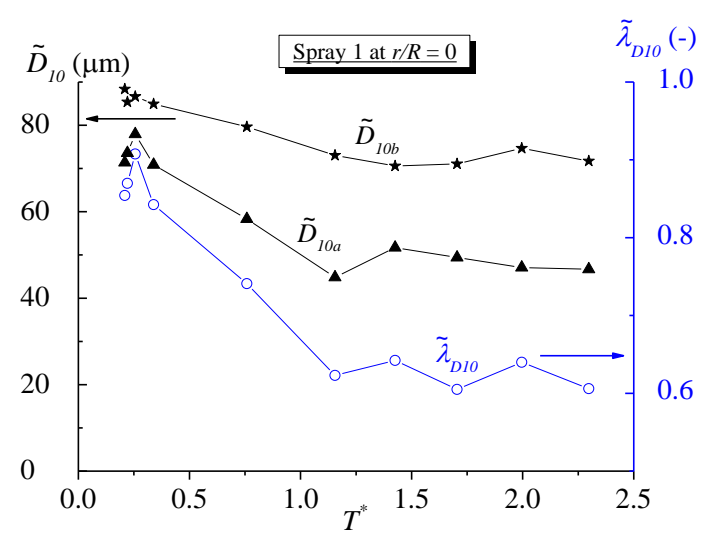

Figure 16: Evolution of the mean droplet diameter before and after impact and of the time averaged droplet size ratio as a function of the dimensionless temperature; spray (1) at $r / R=0$.

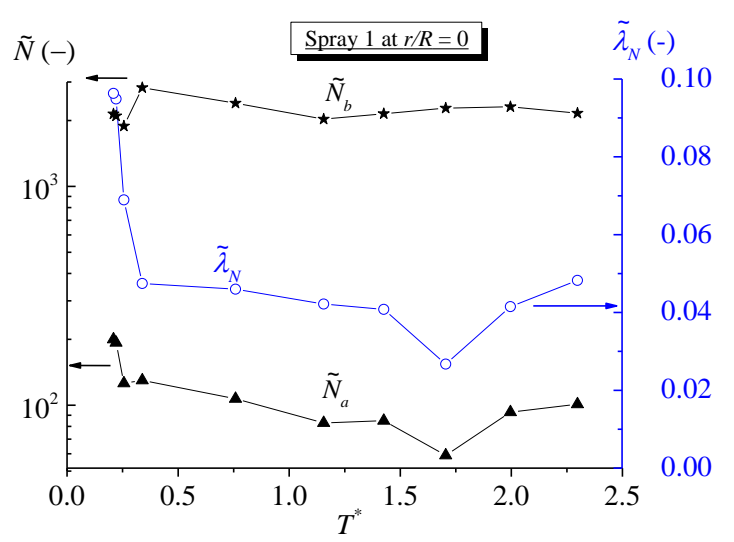

Figure 17: Evolution of the droplet number before and after impact and of the time averaged number ratio as a function of the dimensionless temperature; spray (1) at $r / R=0$.

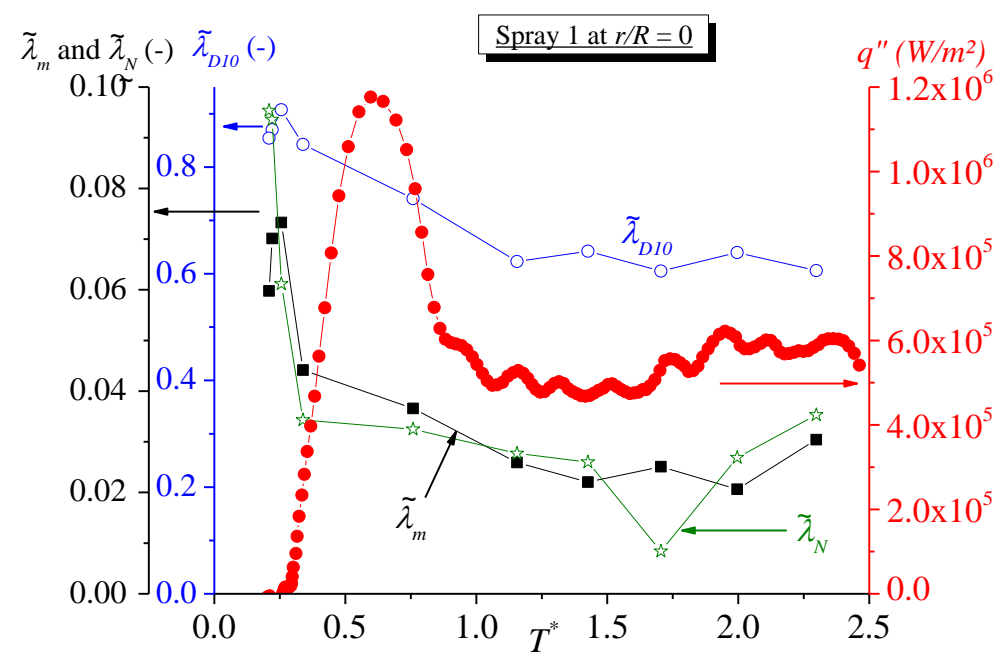

Figure 18: Boiling curve and evolution of the time average droplet size ratio, number ratio and mass ratio during the cooling phase (spray (1) at $X / R=0$ ).

\subsection{Investigation of the secondary spray properties}

In this last section, both time average droplet size and expelled mass ratios are investigated for all 18 tested spray conditions in order to assess the conclusions derived from the deep investigations of the particular case of spray (1). First, the evolution of $\tilde{\lambda}_{D 10}$ and $\tilde{\lambda}_{m}$ along the radius of the disk will be analyse. Then, the influence of the normal Weber number $\widetilde{W} e_{n b}$ will be investigated.

Figures 19 and 20 provide the evolution of $\tilde{\lambda}_{D 10}$ and $\tilde{\lambda}_{m}$ along the disk radius (for the three different $r / R$ positions) and for the three time-average dimensionless temperature intervals. It should be also noted that for a given temperature interval at a given $r / R$ position, $\tilde{\lambda}_{D 10}$ and $\tilde{\lambda}_{m}$ are determined by an average over the six sprays. 
One can observe that the droplet size ratio decreases slightly as $r / R$ increases (Figure 19). This could be related to the smallest droplets which are deviated towards the heated target periphery. Consequently, a larger number of small droplets are detected in this area, leading to a decrease of the average droplet size. Moreover, it appears that for the three $r / R$ positions, the average droplet size increases when the dimensionless temperature decreases. As mentioned before, it is due to the presence of the liquid film expulsing larger droplets. From Figure 20, the expelled mass ratio increases along the disk radius. It is also attributed to the deviation of the smallest droplets that tends to locally increase the mass flux. In addition, the increase of the expelled mass ratio for the three $r / R$ positions confirms the result in Figure 18 at $r / R=0$ when the dimensionless temperature decreases.

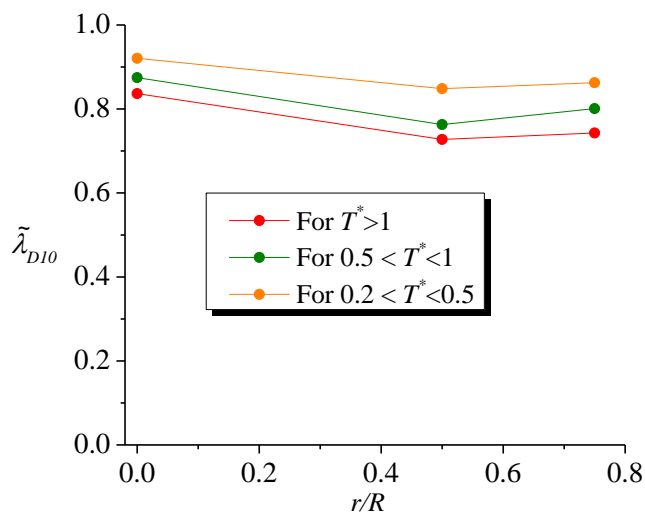

Figure 19: Evolution of the time average droplet size ratio along the radius of the disk for the three dimensionless temperature ranges.

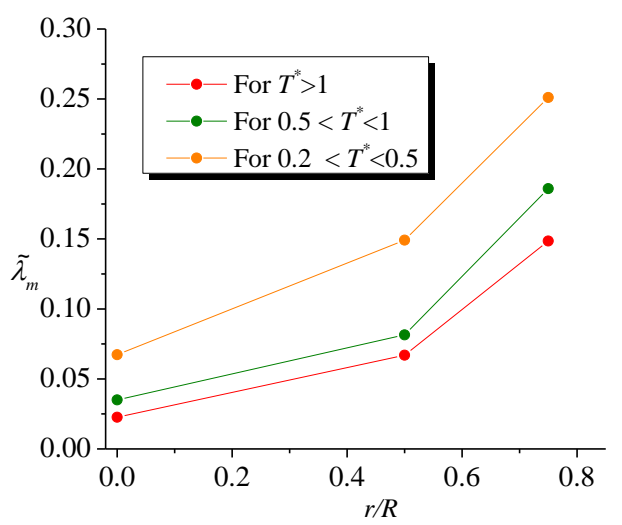

Figure 20: Evolution of the time average mass ratio along the radius of the disk for the three dimensionless temperature ranges.

Figures 21 and 22 describe respectively the evolution of the time average droplet size ratio and the expelled mass ratio as a function of the normal Weber number $\widetilde{W e}_{n b}$ for the three dimensionless temperature intervals and for the 18 spray conditions (i.e. for the six sprays and for the three $r / R$ positions). For the Leidenfrost regime, it seems that the droplet size ratio is quite independent with respect to the normal Weber number while it increases slightly for the two other dimensionless temperatures. Once more, an increase of the size ratio can be noticed when the temperature decreases.

The expelled mass ratio represented in Figure 22 exhibits a strong correlation with the normal Weber number regardless the surface temperature: this ratio decreases according to a power law of the normal Weber number, quantified by:

$\check{\lambda}_{m}=3.35 \widetilde{W} e_{n b}^{-0.85}$

A similar qualitative behaviour was observed by Kalantari and Tropea [21] for impingement of a spray on a cold surface leading to a different empirical correlation. For the current work with a heated wall, no significant effect of the wall temperature on the expelled mass ratio is observed.

The ratio of the mean normal velocity after impact and before impact is plotted in Figure 23 as a function of the mean normal Weber number for the same dimensionless temperatures. The ratio decreases also a power law of the mean normal Weber number:

$\frac{\widetilde{W}_{a}}{\widetilde{W}_{b}}=1.11 \widetilde{W}_{n b}^{-0.38}$ 
This correlation is very close to the one obtained by Kalantari and Tropea [21] (See Figure 23). In addition, it seems that the wall temperature has no significant influence on the velocity of the expelled droplets.

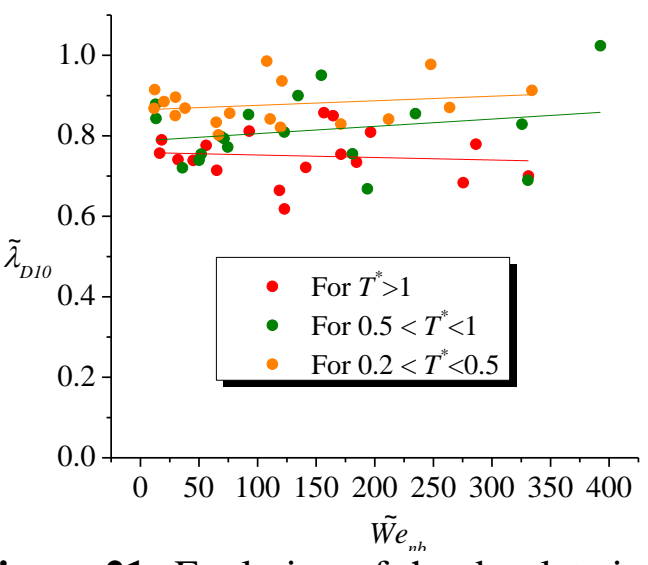

Figure 21: Evolution of the droplet size ratio as a function of the mean normal impact Weber number for the three dimensionless temperature ranges.

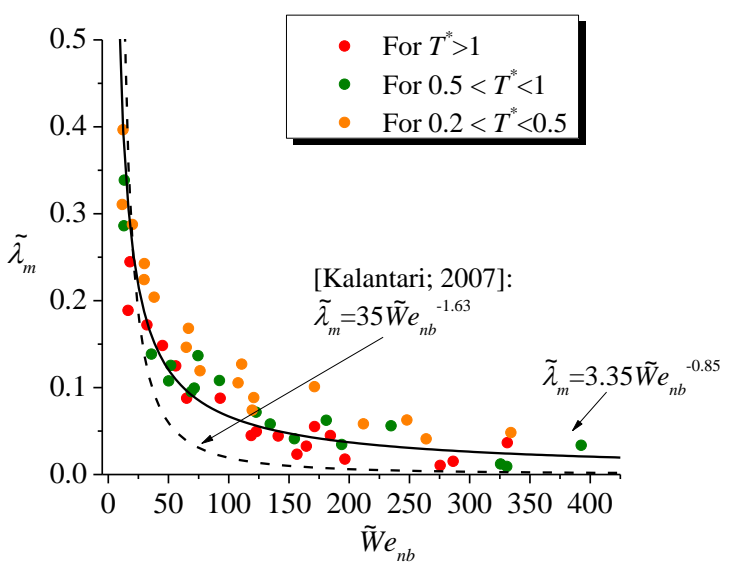

Figure 22: Evolution of the mass ratio as a function of the mean normal impact Weber number for the three dimensionless temperature ranges.

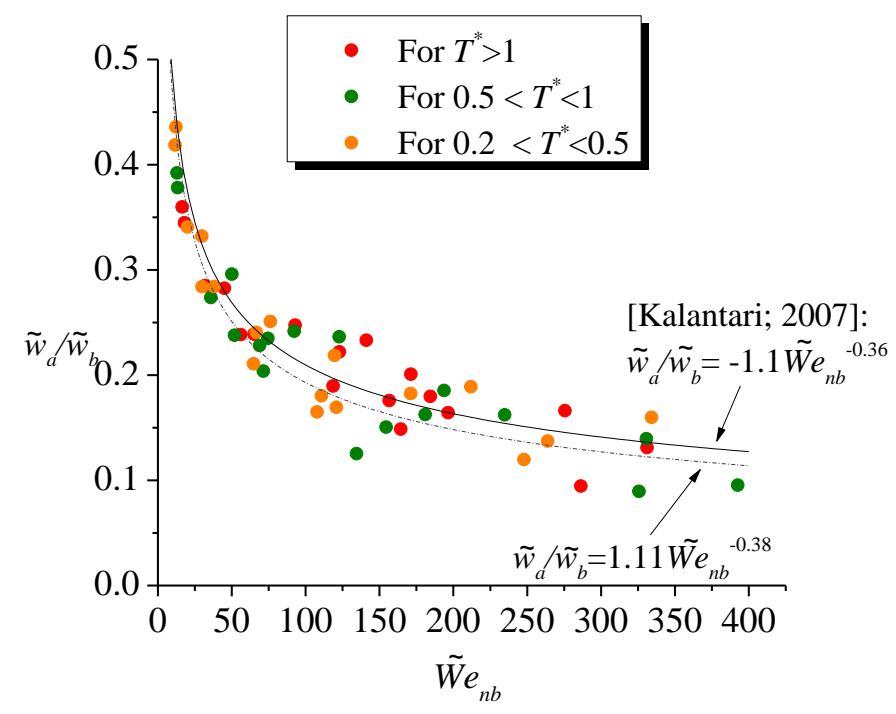

Figure 24: Mean normal velocity ratio of expelled to impinging droplets as a function of the mean normal Weber number for the three dimensionless temperature ranges.

\section{Summary and Conclusions}

This study has highlighted the potential of combining PDA device and infrared thermography measurements to properly describe the impingement of a spray and the characteristics of the secondary droplets during the cooling of a hot surface under conditions close to the ones encountered in industry processes: large target $\left(87.5 \mathrm{~mm}\right.$ radius), surface temperature up to $800^{\circ} \mathrm{C}$ (i.e. well above the Leidenfrost point), liquid mass flux and normal Weber number up to $13.5 \mathrm{~kg} / \mathrm{m}^{2} / \mathrm{s}$ and $330 \mathrm{re-}$ spectively. The main objective of this study was to correlate the characteristics of the secondary spray, the surface temperature and heat flux extracted by the fluid. Phase Doppler and infrared thermography measurements were synchronized in order to measure simultaneously the local characteristics of the droplets, the surface temperature and the subsequent heat flux extracted by the 
water. A set of six different sprays was used and measurements at three locations above the surface were performed in order to analyze a large spectrum of impact conditions (normal Weber number, and impact angle and liquid mass flux). First, by comparing the data obtained in the case of a free spray (i.e. without the disk), the influence of the heated surface on the spray structure was clearly highlighted. The smallest incident droplets, due to their low Stokes number, are deviated toward the disk periphery. This deviation was more pronounced when the surface temperature was lower than the Leidenfrost point. Below this temperature, a vapour flow incoming from the solid surface is generated, due to the strong vaporization of the interacting droplets, leading to a more significant deviation of the smallest incident droplets. Then, the impingement was mainly described by investigating the mean droplet diameter and the mass flux of the secondary sprays. A first result shows that the mean droplet diameter increases when the surface temperature decreases, which could be attributed to the formation of the liquid film potentially generating larger droplets. The expelled mass flux increases also when the surface temperature decreases, but below a given surface temperature, the decrease can be related to a deeper liquid film. Finally, both mean diameter and ejected mass flux ratios present a strong correlation with the normal Weber number.

Future work will concern a further implementation of optical diagnostics to evaluate the contribution of the different heat fluxes involved in energy balance of the impingement of a spray onto a heated surface in order to determine the importance of the heat flux related to liquid vaporization and sensible heat carried by the secondary spray. For that purpose, local spray temperature combined with droplet size and velocity measurements will be required.

\section{References}

[1] C. Mundo, M. Sommerfeld, C. Tropea, Experimental studies of the deposition and splashing of small liquid droplet impinging on a flat surface, in: Proceedings of International Conference on Liquid Atomization and Spraying System, Rouen, France, 1994.

[2] J. Dewitte, P. Berthoumieu, G. Lavergne, An experimental study of droplet hot wall interactions and a survey of the splashing regime, Proceedings of the $5^{\text {th }}$ International Symposium on Multiphase Flow , (2005).

[3] G.E. Cossali, M. Marengo, M. Santini, Single-drop empirical models for spray impact on solid walls: a review, Atomization And Sprays 15 (2005) 699-736.

[4] S. Sivakumar, C. Tropea, Splashing impact of a spray onto a liquid film, Physics Fluids Letter, 14 (2002) L85-L88 .

[5] I.V.Roisman, Tropea, C., Structure of an impinging spray, Proc. 4th International Symposium on Measurements and Techniques for Multiphase Flows, September 10-12, Hangzhou, China (2004).

[6] C.X. Bai, A.D.Gossman, Mathematical modeling of wall films formed by impinging sprays, SAE Tech Paper (1996).

[7] M. Marengo, S. Sikalo, C. Tropea, Impact of drops on inclined dry surfaces, Proc. International Conference on Liquid Atomization and Spraying System, Manchester, United Kingdom, 1998.

[8] R. Schmehl, H. Rosskamp, M. Willmannand, S. Wittig, CFD analysis of spray propagation and evaporation including wall film formation and spray/film, International Journal of Heat and Fluid Flow 20 (1999), 520-529.

[9] J.D. Bernardin, I.Mudawar, Film boiling heat transfer of droplets streams and sprays, International Journal of Heat and Mass Transfer 40 (11) (1997) 2579- 2593.

[10] M.A. Gonzales, G.L. Borman, R.D. Reitz, A study of diesel cold starting using both cycle analysis and multidimensional calculations, SAE Tech Paper 910180 (1991).

[11] A.P. Watkins, D.M. Wang, A new model for diesel spray impaction on wall and comparison with experiments, Proceeding Comodia (1990) 243-248. 
[12] K., Park, A.P. Watkins, Comparison of wall spray impaction models with experimental data on drop velocities and sizes. International Journal of Heat and Fluid Flow 17 (1996) 424-438.

[13] A. Moita, A. Moreira, Development of empirical correlations to predict the secondary droplet size of impacting droplets onto heated surfaces, Experiments in Fluids 47 (2009) 755-768.

[14] A. Moita, A. Moreira, Drop impacts onto cold and heated rigid surfaces, International Journal of Heat and Fluid Flow 28:735-752 (2007).

[15] J.D. Naber, P. Farrel, Hydrodynamics of droplet impingement on a heated surface, SAE Tech Paper 930919 (1993).

[16] S.W. Akhtar, A .J. Yule, Droplet Impaction on a heated surface at high Weber numbers Proceedings of International Conference on Liquid Atomization and Sprays System (2001) Zurich, Switzerland.

[17] G. Castanet, T. Liénart, F. Lemoine, Dynamics and temperature of droplets impacting onto a heated wall, International Journal of Heat and Mass Transfer 52 (2009) 670-679.

[18] G., Castanet, P. Dunand, O. Caballina, F. Lemoine, High-speed shadow imagery to characterize the size and velocity of the secondary droplets produced by drop impacts onto a heated surface, Experiments in Fluids 54 (2013) 1489-1507.

[19] S.W. Park and C.S. Lee, Macroscopic and microscopic characteristics of a fuel sprayimpinged on the wall, Experiments in Fluids 54 (2004) 1489-1507.

[20] M.R.O. Panao, A. Moreira, Flow characteristics of spray impingement in PFI injection systems, Experiments in Fluids 39 (2005) 364-374.

[21] Kalantari, D. and Tropea, C., Spray impact onto flat and rigid walls: Empirical characterization and modeling, International Journal of Multiphase Flow 33 (2007) 525-544.

[22] K.A. Estes, I. Mudawar, Correlation of Sauter mean diameter and critical heat flux for spray cooling of small surfaces, International Journal of Heat and Mass Transfer 38 (1995) 29852996.

[23] Al-Ahmadi H.M., Yao, S.C., Spray cooling of high temperature metals using high mass flux industrial nozzles, Experimental Heat Transfer 21 (2008) 38-51.

[24] Cox T.L., Yao S.C., Heat transfer of sprays of large water drops impinging on high temperature surfaces, Journal of Heat Transfer 121 (2) (1999) 446-450.

[25] Jia, W. and Qiu, H.H., Experimental investigation of droplet dynamics and heat transfer in spray cooling, Experimental Thermal and Fluids Science 27 (2003) 829-838.

[26] Labergue, A., Gradeck, M., Lemoine, F., Comparative study of the cooling of a hot temperature surface using sprays and liquid jets, International Journal of Heat and Mass Transfer 81 (2015) 889-900.

[27] Gradeck, M., Ouattara, J.A , Rémy, B., Maillet, D., Solution of an inverse problem in the hankel space - infrared thermography applied to estimation of a transient cooling flux, Experimental Thermal and Fluids Science 36 (2012) 56-64.

[28] Ouattara, A., Gradeck, M., Maillet, D., Remy, B., Solution of IHCP estimation of a non uniform source using an inverse heat conduction method based on an analytical solution, Proceedings of Eurotherm (2008).

[29] Maillet D., André S., Batsale J.C., Degiovanni A., Moyne C., Thermal quadrupoles : solving the heat equation through integral transforms, John Wiley \& Sons Ed (2000).

[30] Yao S. C., Cox T.L. A general heat transfer correlation for impacting water sprays on hightemperature surfaces, Experimental Heat Transfer 15 (2002) 207-219.

[31] Pederson C.O., An experimental study of the dynamic behavior and heat transfer characteristics of water droplets impinging upon a heated surface, International Journal of Heat and Mass Transfer 13 (1970) 369-381. 